\title{
Circulating bacterial signature is linked to metabolic disease and shifts with metabolic alleviation after bariatric surgery
}

Rima M. Chakaroun ${ }^{1 *+} \mathbb{D}$, Lucas Massier ${ }^{1,2 \dagger}$, Anna Heintz-Buschart ${ }^{3,4}$, Nedal Said ${ }^{1,5}$, Joerg Fallmann ${ }^{6}$, Alyce Crane ${ }^{1}$, Tatjana Schütz ${ }^{1}$, Arne Dietrich ${ }^{7}$, Matthias Blüher ${ }^{1,8}$, Michael Stumvoll ${ }^{1}$, Niculina Musat ${ }^{5}$ and Peter Kovacs ${ }^{1,9}$

\begin{abstract}
Background: The microbiome has emerged as an environmental factor contributing to obesity and type 2 diabetes (T2D). Increasing evidence suggests links between circulating bacterial components (i.e., bacterial DNA), cardiometabolic disease, and blunted response to metabolic interventions. In this aspect, thorough next-generation sequencing-based and contaminant-aware approaches are lacking. To address this, we tested whether bacterial DNA could be amplified in the blood of subjects with obesity and high metabolic risk under strict experimental and analytical control and whether a putative bacterial signature is related to metabolic improvement after bariatric surgery.

Methods: Subjects undergoing bariatric surgery were recruited into sex- and BMI-matched subgroups with $(n=24)$ or without T2D $(n=24)$. Bacterial DNA in the blood was quantified and prokaryotic 165 rRNA gene amplicons were sequenced. A contaminant-aware approach was applied to derive a compositional microbial signature from bacterial sequences in all subjects at baseline and at 3 and 12 months after surgery. We modeled associations between bacterial load and composition with host metabolic and anthropometric markers. We further tested whether compositional shifts were related to weight loss response and T2D remission. Lastly, bacteria were visualized in blood samples using catalyzed reporter deposition (CARD)-fluorescence in situ hybridization (FISH).
\end{abstract}

Results: The contaminant-aware blood bacterial signature was associated with metabolic health. Based on bacterial phyla and genera detected in the blood samples, a metabolic syndrome classification index score was derived and shown to robustly classify subjects along their actual clinical group. T2D was characterized by decreased bacterial richness and loss of genera associated with improved metabolic health. Weight loss and metabolic improvement following bariatric surgery were associated with an early and stable increase of these genera in parallel with improvements in key cardiometabolic risk parameters. CARD-FISH allowed the detection of living bacteria in blood samples in obesity.

Conclusions: We show that the circulating bacterial signature reflects metabolic disease and its improvement after bariatric surgery. Our work provides contaminant-aware evidence for the presence of living bacteria in the blood and suggests a putative crosstalk between components of the blood and metabolism in metabolic health regulation.

Keywords: Blood bacteria, Obesity, Metabolic syndrome, Type 2 diabetes, Bariatric surgery

\footnotetext{
* Correspondence: rima.chakaroun@medizin.uni-leipzig.de

${ }^{\dagger}$ Rima M. Chakaroun and Lucas Massier contributed equally to this work.

${ }^{1}$ Medical Department III - Endocrinology, Nephrology, Rheumatology,

University of Leipzig Medical Center, Leipzig, Germany

Full list of author information is available at the end of the article
}

C The Author(s). 2021 Open Access This article is licensed under a Creative Commons Attribution 4.0 International License, which permits use, sharing, adaptation, distribution and reproduction in any medium or format, as long as you give appropriate credit to the original author(s) and the source, provide a link to the Creative Commons licence, and indicate if changes were made. The images or other third party material in this article are included in the article's Creative Commons licence, unless indicated otherwise in a credit line to the material. If material is not included in the article's Creative Commons licence and your intended use is not permitted by statutory regulation or exceeds the permitted use, you will need to obtain permission directly from the copyright holder. To view a copy of this licence, visit http://creativecommons.org/licenses/by/4.0/. The Creative Commons Public Domain Dedication waiver (http://creativecommons.org/publicdomain/zero/1.0/) applies to the data made available in this article, unless otherwise stated in a credit line to the data. 


\section{Background}

The major health burden of obesity is largely attributable to its associated diseases such as type 2 diabetes (T2D), the metabolic syndrome (MetS), and cardiovascular diseases. Along known risk factors, such as genetic predisposition, poor diet, and lower physical activity, shifts in the gut microbial composition have been shown to contribute to metabolic inflammation at the advent of obesity and T2D [1-5]. Beyond composition, low gut bacterial diversity has been associated with increased obesity, insulin resistance, dyslipidemia, and increased inflammation [6, 7]. More recently, low gut bacterial load has been identified as a key driver related to chronic inflammatory states such as Crohn's disease [8]. Converging evidence further points to an important role of the gut microbiome as a therapeutic target and prognostic marker: Weight loss interventions, such as diet and bariatric surgery, profoundly affect the gut microbiota composition, leading to an increased bacterial gene count and bacterial richness. This, in turn, is associated with a decrease in inflammatory markers and an increase in insulin sensitivity up to complete remission of T2D [9-11]. Accordingly, weight loss interventions are less effective in improving insulin resistance and inflammatory state in patients with low bacterial gene diversity [7].

Although most evidence has been submitted for the gut microbiome, host tissues-including the blood [12], liver, and adipose tissue [13, 14]-have been shown to accommodate microbial consortia finally accessible through culture-independent techniques. Few studies further linked increased bacterial DNA load in the circulation with the incidence of T2D $[15,16]$ and cardiovascular disease [17]. Moreover, bacterial signature in the blood has been linked to the circulatory compartment it derives from (i.e., systemic vs portal circulation) [18], systemic inflammation [18], T2D presence [13, 14, 19], and metabolic disease severity [20]. Similarly, there is mounting evidence for the diagnostic application of circulating bacterial DNA highlighted in a recent work employing contaminant-aware approaches to show that it can discriminate between multiple types of cancer and is highly dependent on disease severity [21]. On the other hand, only one study contemplated the interplay between metabolic interventions and circulating bacterial markers: Subjects with an established bacterial DNA translocation based on qPCR detection did not experience a remission of $\mathrm{T} 2 \mathrm{D}$ or significant improvement in insulin sensitivity despite significant weight loss after bariatric surgery [22]. These results advocate a closer look at blood-borne bacterial DNA, bacterial signatures and their role in health and disease.

It is worth noting that studies on bacterial load and composition in low bacterial biomass environments such as the blood are subject to technical biases including highly underestimated environmental contamination [23]. To our knowledge, only few studies have actively controlled for contamination so far $[13,14,21]$, and only two included a bioinformatic contaminant-aware approach $[13,14]$. We therefore applied a contaminationaware approach to test the hypothesis that the presence, composition, and load of bacterial DNA in the blood reflect obesity, metabolic risk factors, and inflammatory burden as well as weight loss associated changes in anthropometric and metabolic parameters after bariatric surgery.

\section{Methods \\ Characterization of study participants and sample collection}

Sixty-four subjects were recruited at the University of Leipzig Medical Center, Germany, after matching for BMI and sex differing on T2D status with 32 subjects having no T2D and 32 with T2D according to ADA criteria [24]. However, 16 subjects had to be excluded from longitudinal comparisons due to missing follow-up samples in at least one timepoint $(n=15)$ or missing followup phenotypes $(\mathrm{n}=1)$. If not indicated otherwise, 48 subjects (24 with T2D, 24 without) were included in all analyses (Table 1-baseline cohort characteristics of subjects with complete follow-up $(n=48)$, Additional file 1 : table S1-baseline cohort characteristics with initial matching $(n=64)$ ). Individuals fulfilled the following inclusion criteria: (1) eligibility to bariatric surgery according to international clinical guidelines $\left(B M I \geq 40 \mathrm{~kg} / \mathrm{m}^{2}\right.$, or $\geq 35 \mathrm{~kg} / \mathrm{m}^{2}$ with at least one obesity-associated metabolic disease) and internal clinical multidisciplinary panel, (2) no chronic or acute inflammatory disease as determined by blood cell counts and CRP or clinical signs of infection, (3) no evidence of coronary or peripheral artery disease, (4) no known thyroid dysfunction, (5) no antibiotics intake in the 3 months prior to the study visit, (6) no pregnancy or nursing, and (7) no NSAID intake within $78 \mathrm{~h}$ prior to the study visit.

Subjects were extensively phenotyped before bariatric surgery and at month 3 and 12 post-bariatric surgery. Phenotyping included clinical and anthropometric assessment in conjunction with the evaluation of patient medical history and physical examination. Among other parameters, blood pressure, waist and hip circumference measurements, and bioimpedance measurement for body composition analysis, including automatic calculation of visceral fat rating (BC-418 MA, Tanita, Tokyo, Japan), were assessed. Obesity-associated diseases such as T2D [24], hypertension [25], and hyperlipidemia [26] were defined according to the medical association definition of the specific disease. Metabolic syndrome (MetS) was defined according to international conventions [27]. Excess BMI loss (EBL) was calculated as follows: EBL = 
Table 1 Baseline cohort characteristics of subjects with complete follow-up

\begin{tabular}{|c|c|c|c|}
\hline Baseline characteristics & NGT & T2D & P-value \\
\hline $\mathbf{N}$ & 24 & 24 & \\
\hline \multicolumn{4}{|l|}{ General } \\
\hline Sex (F/M) & $18 / 6$ & $17 / 7$ & 1 \\
\hline Age (years) & $46.8 \pm 8.59$ & $50.1 \pm 7.94$ & 0.007 \\
\hline BMI $\left(\mathrm{kg} / \mathrm{m}^{2}\right)$ & $50.8 \pm 6.59$ & $49.0 \pm 6.68$ & 0.943 \\
\hline WHR & $0.91[0.87 ; 0.95]$ & $0.98[0.99 ; 1.03]$ & 0.006 \\
\hline GFR $\left(\mathrm{ml} / \mathrm{min} / 1.73 \mathrm{~m}^{2}\right)$ & $93.8[80.6 ; 114]$ & $94.0[79.6 ; 110]$ & 0.996 \\
\hline \multicolumn{4}{|l|}{ Smoking status } \\
\hline Active smokers, N (\%) & $2(8.70 \%)$ & $3(12.5 \%)$ & 1 \\
\hline \multicolumn{4}{|c|}{ Glycemia, insulin resistance, and antidiabetic medication intake } \\
\hline HbA1c (\%) & $5.38[5.29 ; 5.58]$ & $6.56[5.95 ; 8.22]$ & $<0.001$ \\
\hline FPG (mmol/l) & $5.25[4.98 ; 5.42]$ & $7.42[6.54 ; 11.7]$ & $<0.001$ \\
\hline HOMA-IR & $3.10[2.27 ; 4.53]$ & $8.59[5.71 ; 13.8]$ & $<0.001$ \\
\hline Nr antidiabetics & $0.00[0.00 ; 0.00]$ & $1.00[1.00 ; 3.00]$ & $<0.001$ \\
\hline \multicolumn{4}{|c|}{ Hypertension status and antihypertensive medication } \\
\hline Hypertension, $\mathrm{N}(\%)$ & $16(66.7 \%)$ & $22(91.7 \%)$ & 0.076 \\
\hline Number of anti-HTN drugs, $\mathrm{N}$ & $1.00[1.00 ; 3.00]$ & $3.50[1.00 ; 5.00]$ & 0.067 \\
\hline Systolic blood pressure $(\mathrm{mmHg})$ & $131 \pm 11.0$ & $133 \pm 14.7$ & 0.665 \\
\hline Diastolic blood pressure $(\mathrm{mmHg})$ & $76.8 \pm 8.26$ & $76.4 \pm 15.1$ & 0.924 \\
\hline \multicolumn{4}{|c|}{ Dyslipidemia and antihyperlipidemic medication intake } \\
\hline LDL-C (mmol/l) & $3.25 \pm 0.87$ & $3.09 \pm 0.93$ & 0.545 \\
\hline HDL-C (mmol/l) & $1.27[1.02 ; 1.52]$ & $1.10[0.94 ; 1.30]$ & 0.105 \\
\hline TG $(\mathrm{mmol} / \mathrm{l})$ & $1.52(0.69)$ & $1.67[1.42 ; 2.24]$ & 0.017 \\
\hline Statin, N (\%) & $1(4.17 \%)$ & $7(29.2 \%)$ & 0.048 \\
\hline Ezetimib, N (\%) & $0.00(0.00 \%)$ & $2(8.33 \%)$ & 0.489 \\
\hline \multicolumn{4}{|l|}{ Blood and inflammatory markers } \\
\hline Hemoglobin (g/dl) & $13.4[13.0 ; 14.5]$ & $14.2[13.4 ; 14.9]$ & 0.115 \\
\hline Leukocytes (Gpt/l) & $6.70[5.25 ; 7.20]$ & $8.30[7.65 ; 10.7]$ & $<0.001$ \\
\hline $\mathrm{CRP}(\mathrm{pg} / \mathrm{ml})$ & $10.4[3.47 ; 17.9]$ & $8.72[5.24 ; 22.8]$ & 0.546 \\
\hline
\end{tabular}

Median and first, as well as third quartile limits (median [q1; q3]) are shown for non-normally distributed, continuous variables. For normally distributed, continuous variables, data are given in mean \pm standard deviation (mean \pm SD). For categorical parameters, total numbers (percentage) are shown. Significant $\mathrm{p}$ values are depicted in bold. Abbreviations: BMI body mass index, CRP C-reactive protein, FPG fasting plasma glucose, HbA1c glycated hemoglobin A1c, HDL highdensity lipoprotein, HOMA-IR homeostatic model assessment for insulin resistance, $L D L$ low-density lipoprotein, NGT normal glucose tolerance, $T 2 D$ type 2 diabetes, TG triglycerides, WHR waist to hip ratio

$\left(\left(\mathrm{BMI}_{\text {baseline }}-\mathrm{BMI}_{12 \text { months }}\right) / \mathrm{BMI}_{\text {baseline }}-25\right) \times 100[28]$, and poor response was defined as an EBL $<50 \%$, whereas a good response was defined as an EBL > 60\% (adapted from [28]) (Additional file 1: table S2-phenotypes of good vs poor responders).

\section{Replication cohort}

To validate results from bacterial quantification and associations thereof with host parameters, data from a subgroup consisting of 62 subjects belonging to a previously described cohort from the same center were analyzed (Additional file 1: Table S3-phenotype replication cohort). Phenotyping procedures and cohort description of the whole cohort are available elsewhere [14]. In brief, patients are part of a larger study including 75 subjects with obesity undergoing laparoscopic RYGB and were recruited at the University of Leipzig Medical Center, Germany. Exclusion criteria were the same as for the primary cohort. Subjects received clinical phenotyping consisting of oral glucose tolerance test, collection of anthropometric (age, sex, and body mass index (BMI)) and metabolic parameters such as fasting plasma glucose (FPG), fasting plasma insulin (FPI), high- and lowdensity lipoprotein (HDL, LDL) cholesterol, homeostasis model assessment for insulin resistance (HOMA-IR), and hemoglobin A1c $(\mathrm{HbA} 1 \mathrm{c})$. Moreover, blood cell 
counts and hsCRP were measured in local routine laboratory measurements. Tumor necrosis factor alpha (TNF- $\alpha$ ) and interleukin 6 (IL-6) were measured by high-sensitive ELISA (R\&D Systems, Minneapolis; HSTA00E and HS600B) according to the manufacturer's protocol.

\section{Sample preparation}

Blood samples were collected after an overnight fast at each timepoint, and EDTA blood samples were collected for DNA isolation and stored at $-20^{\circ} \mathrm{C}$. FPG was measured using the hexokinase method, HDL and LDL cholesterol were measured using enzymatic assays, and FPI was measured using chemiluminescence assay according to standard laboratory procedures. C-reactive protein (CRP) was measured using an Image Automatic Immunoassay System (Beckman Coulter). All measurements were performed according to routine laboratory procedures. HOMA-IR was calculated as previously described [29]. Lipopolysaccharide binding protein (LBP) was measured using the HK315 HUMAN LBP ELISA Kit (Hycult Biotech, Uden, Netherlands) according to the manufacturer's recommendations. Samples and follow-up availability dictated the sample size used for analyses at each step as illustrated in Additional file 1: Fig. S1.

\section{Bacterial DNA extraction, quantification, and amplification for sequencing \\ Bacterial DNA extraction}

To minimize contamination, all steps were performed under a sterile class II laminar flow hood using aseptic measures including the use of one-way lab coats, elbowlength gloves, and facemasks. All non-organic liquids and required materials were subjected to at least 120 min of UV radiation. Furthermore, negative controls consisting of UV-treated PBS (filled in EDTA vials using venipuncture ware from the same batch to simulate venous puncture) were included and carried through all experimental procedures, including sequencing.

DNA was extracted from $200 \mu \mathrm{l}$ whole EDTA blood using the QIAMP Blood MiniKit (Qiagen, Germany) following the manufacturer's recommendations with an additional lysozyme step at $37^{\circ} \mathrm{C}$ overnight $(0.25 \mathrm{mg} / \mathrm{ml}$, L7615, Sigma-Aldrich, MO, USA). DNA yield, integrity, and quality were assessed using Quant-iT PicoGreen dsDNA kit (Invitrogen, CA, USA) and Nanodrop 2000 spectrometer (Thermo Fisher Scientific, MA, USA).

\section{Quantification of bacterial 16S rRNA gene}

Bacterial DNA was quantified by qPCR amplification of the bacterial 16S rRNA gene using previously published primers (F_Bact 1369: 5' -CGGTGAATACGTTCCCGG$3^{\prime}$ and R_Prok 1492:5' -TACGGCTACCTTGTTACG
ACTT-3') [17, 30, 31]. All qPCR reactions were performed in duplicate each, using $50 \mathrm{ng}$ of whole extracted DNA, prepared in a PCR clean room, and run on a LightCycler 480 (Hoffmann-La Roche, Basel, Switzerland) with the following conditions: $50^{\circ} \mathrm{C}$ for 2 min, $95^{\circ} \mathrm{C}$ for $10 \mathrm{~min}$ and 40 cycles of denaturation at $95^{\circ} \mathrm{C}$ for $15 \mathrm{~s}$, annealing at $60^{\circ} \mathrm{C}$ for $30 \mathrm{~s}$, and extension at $72{ }^{\circ} \mathrm{C}$ for $30 \mathrm{~s}$. The amount of amplified bacterial DNA was calculated using mean $\mathrm{Cp}$ values for each duplicate against a standard curve from E. coli JM 109 DNA dilutions (Promega, MA, USA), which included seven duplicates ranging from $1.25 \mathrm{fg}$ to $0.2 \mathrm{ng}$ total bacterial DNA. Quantification of the analyzed samples was performed in three runs with $\mathrm{R}^{2} \geq 0.995$ and a mean PCR efficiency of $2.06 \pm 0.03$. Analysis was conducted according to the Livak method [32]. Results were in concordance with a commercially available kit (Zymoresearch, CA, USA) $(\mathrm{n}=13, \mathrm{r}=0.74, \mathrm{p}=0.004)$ and proved more sensitive $(\Delta \mathrm{Ct}=3.9)$. Obtained concentrations were normalized to the total concentration of extracted DNA as well as the used blood volume and are given as pg bacterial DNA per $\mu \mathrm{g}$ of isolated DNA. This is due to the variation of amounts of total extracted DNA from the same $200 \mu$ l blood volume for each sample, which ranged from 12.7 to $98.2 \mathrm{ng} / \mu \mathrm{l}$. Blood bacterial quantity in the replication cohort was independently measured according to the same protocol. To overcome inter-assay variability, blood bacterial load was standardized ( $\mathrm{z}$-score transformation) within the cohorts and analyses were conducted on standardized bacterial quantities for each cohort separately as well as both of them combined.

\section{5 rRNA gene sequencing analysis}

After extensive testing of combinations of primers and polymerases for amplification of prokaryotic/bacterial 16S rRNA variable regions [14], V4-V5 primers adapted from the Ribosomal Database Project (RDP) (V4-F: 5' ACTGGGCGTAAAGCG-3'; V5-R: 5' -CCGTCAATTC CTTTGAGTTT-3') were used [33]. PCR reactions were prepared in triplicate in a sterile laminar flow hood and performed using $50 \mathrm{ng}$ total DNA in a total reaction volume of $25 \mu \mathrm{l}$ containing $1.25 \mu \mathrm{l}$ of each primer $(10 \mu \mathrm{M})$, $12.5 \mu \mathrm{l} 2 \mathrm{x}$ Q5 Reaction Buffer (including $\mathrm{Q}^{\circ}$ HighFidelity DNA Polymerase, New England BioLabs, MA, USA), and $6.25 \mu \mathrm{l}$ UV-ed PCR-grade water. The reaction was carried out on a LightCycler 480 (Hoffmann-La Roche, Basel, Switzerland) under the following conditions: $98^{\circ} \mathrm{C}$ for $2 \mathrm{~min}$ and 40 cycles of denaturation at $98^{\circ} \mathrm{C}$ for $30 \mathrm{~s}$, annealing at $58^{\circ} \mathrm{C}$ for $30 \mathrm{~s}$, and extension at $72{ }^{\circ} \mathrm{C}$ for $30 \mathrm{~s}$. Each PCR reaction included at least 3 negative controls in addition to the extraction controls (blank controls) and the absence of detectable PCR products in these negative controls was confirmed by gel 
electrophoresis. Regardless of this, negative controls for each run were pooled and carried through sequencing to be used for contaminant identification. Replicate amplicons were pooled and purified using Agencourt Ampure magnetic purification beads (Beckman Coulter Indianapolis, IN, USA) according to the manufacturer's protocol to remove short amplification products and primer dimers. DNA amounts were quantified using Quant-iT PicoGreen dsDNA kit (Invitrogen, CA, USA). Library preparation and paired-end sequencing were performed commercially (BGI Genomic, Shenzhen, China) on Illumina Hiseq2500 technology and using custom fusion primers in a one-step PCR approach.

\section{Bioinformatics and statistical analyses Statistical analyses}

Statistical analyses were performed in R v3.5.0 (R Development Core Team, 2008). Prior to statistical testing, distribution and single test assumptions were checked to ensure the test's suitability. For cross-sectional comparisons between two groups, the Wilcoxon signed-rank test was used for non-normally distributed variables according to Shapiro-Wilk testing, whereas unpaired Student's t-test was used to compare normally distributed variables. Median and first as well as third quartile limits are depicted for non-normally distributed variables, whereas for normally distributed continuous variables, data are given as mean \pm standard deviation (SD). The Friedman test was used to compare dependent groups at different timepoints. Categorical parameters were analyzed using the chi-square test. Bivariate correlation analyses were performed using Spearman's rank correlation test. Figures were generated using ggplot2 [34] v3.1.0, ggpubr v0.2 [35], and corrplot v.0.84 [36] as well as phyloseq v1.26.1 [37] and relabeled in Adobe Illustrator (Adobe Inc., CA, USA). A p-value threshold of 0.05 was used to depict statistical significance. Analyses were adjusted for multiple hypotheses ad modum Benjamini-Hochberg, in which case a false discovery rate (FDR) of Padj $<0.05$ was considered significant.

\section{Processing of $16 \mathrm{~S}$ rRNA reads and amplicon sequence variant (ASV) clustering}

A total of 12,857,649 Illumina Hiseq quality filtered paired-end reads were obtained from BGI, with samples having a median read count of 63,055 [9661-64,630] [38]. Preprocessing and quality filtering consisted of removing reads with a certain proportion of low-quality (20) bases (20\% of read original length), contaminated by adapter ( 5 bases overlapped by reads and adapter with maximal 3 bases mismatch allowed), with ambiguous bases and with low complexity (reads with 10 consecutive same base). Subsequently, quality was checked using Multiqc [39] and data was imported to QIIME2
[40] V2019.1 for analysis [38, 41]. Denoising, dereplication, merging, and chimera filtering as well as ASV inference were done in one step using the DADA2 [42] plugin in QIIME 2, resulting in $40269 \pm 6033$ nonchimeric reads with an average read length of $329 \mathrm{bp}$. Reads were truncated at a length of $242 \mathrm{bp}$. For phylogenetic analyses, primer-truncated and quality trimmed reads were used to create an alignment using mafft [43]. The alignment was masked to remove uninformative highly variable regions and a rooted tree was generated using the align-to-tree-mafft-fasttree plugin. For taxonomic classification, a scikit-learn [44] naive Bayes classifier was created against the taxonomic classification from ARB-SILVA [45] 132 release (99\% OTU data set), which was trained for the used primers. Only reads mapped to bacterial taxonomy were retained. This resulted in a total of 2860 bacterial ASVs with a total frequency of 7,784,509. Derived taxonomy, tree, and feature table were imported into phyloseq [37] v1.26.1, and all subsequent analyses were performed in $\mathrm{R} v$ 3.5.0 0 (R Development Core Team, 2008).

\section{Bacterial contaminant identification in blood samples}

A bioinformatic decontamination step using the Decontam [46] package v1.2.1, which is intended for the identification of contaminants in low bacterial biomass samples, was added. To identify contaminant ASVs, the "prevalence" method was applied. A binomial distribution with low scores for low prevalence ASVs was found around 0.1 and high prevalence ASVs with higher confidence increasing around 0.175 , which led us to select a data-driven contaminant score of 0.175 instead of the default classification score 0.1 (Additional file 1: Fig. S2). Using these parameters, 114 features were classified as potential contaminants and removed, leading to a total of 2746 ASVs (Additional file 2: Table S2, Additional file 2: Table S3, Additional file 2: Table S4). The pruned phyloseq object was then used for subsequent analyses. We moreover added a more stringent decontamination step using a contaminant score of 0.5 leading to flagging of each of the 172 ASVs appearing in the negative samples as contaminants. While ASVs derived from both decontamination scores led to similar results in associations of taxonomy with host variables (data not shown), the data-driven decontamination at a lower score was more likely to influence differential abundance analyses. This led us to use a more stringently decontaminated taxonomy while performing differential abundance testing to avoid spurious observations. The stringently decontaminated taxonomy included a total of 2688 ASVs (Additional file 2: Table S5, Additional file 2: Table S6). 


\section{Analyses of bacterial composition and its association with host phenotypes}

Alpha diversity measures (Shannon diversity, observed richness) were calculated and RDA on Bray-Curtis-dissimilarity distances was conducted to identify host covariates contributing to bacterial community composition in the blood in vegan [47] v2.5-4. Samples with missing observations (NAs) in the covariates were eliminated from the dataset prior to analyses. Variables used in the RDA model included T2D status, sex, metformin use, PPI use, timepoint, MetS status, diabetes alleviation after surgery, EBL, surgical procedure, BMI, systolic blood pressure, eGFR, CRP, HbA1c, HDL cholesterol, LDL cholesterol, triglycerides, total fat mass in percentage, visceral fat ratio, number of antidiabetics, number of antihyperlipidemic drugs, number of antihypertensive drugs, white blood cell count, age, bacterial load, and waist to hip ratio. We further used automatic stepwise modeling and model selection using the ordistep approach both ways with 999 permutations to identify the most significant covariates contributing to community composition. Microbiome [48] v1.4.2. was used to perform correlation analyses between relative taxa abundances and host variables of interest applying Spearman's rank correlation.

A random forest classification (randomForest [49] v4.6-14) approach on both phylum- and genus-level abundances was used to develop classification indices for MetS for subjects at baseline. For phylum- and genuslevel, NGS -derived read counts from MetS and nonMetS subjects were normalized by the total read count of the corresponding phylum or genus and used as features for training of a random forest classifier. The classification index predicted ranges between 0 and 1 and corresponds to the out-of-bag predicted probability of being classified as belonging to the class of interest (i.e., having MetS) with higher scores indicating higher probability for having MetS. Performance of classification indices was quantified using receiver operating characteristic curve and AUC using pROC [50] v1.14.0. Cross validation was performed using the rfUtilities package [51, 52] v2.1-5. Specifics of random forest classification are as follows: number of trees 500; number of variables tried at each split 11; OOB estimate of error rate at genus level 21.3\%, at phylum level 29.8\%; classification accuracy for model at genus level: user accuracy 100, producer accuracy 79.1, model kappa $=0.147$, model OOB error $=0.204$, model error variance $=4.7 \times 10^{-5}$; and classification accuracy for model at phylum level: user accuracy 91.2, producer accuracy 79.5, model kappa $=0.1357$, model OOB error 0.25 , model error variance $=$ $7.7 \times 10^{-4}$.

Differential abundance analyses for preassigned dichotomous groups were performed using DESeq2 [53] v1.22.2. Size factors for count data were calculated using the poscounts estimator. Dispersion estimates were performed using the DESeq command, a local regression model was used to fit and test the data, and the negative binomial Wald test was used to test differential abundance (log2 fold change) and significance. Results are reported for differentially abundant taxa with a significance Padj $<0.01$ after multiple hypotheses correction ad modum Benjamini-Hochberg. Specifically, for the comparison between $\mathrm{T} 2 \mathrm{D}$ remission and no remission following bariatric surgery, only subjects with T2D at baseline were retained in the analyses (Additional file 1: Fig. S3).

\section{Catalyzed reporter deposition (CARD)-fluorescence in situ hybridization (FISH) \\ Blood sample collection and cell separation}

To visualize and quantify bacteria in the blood, samples from one patient post-bariatric surgery (male, 38 years, currently 60 months post-bariatric surgery, current BMI $30 \mathrm{~kg} / \mathrm{m}^{2}$, no T2D, no MetS, initial BMI $61.9 \mathrm{~kg} / \mathrm{m}^{2}$, EBL $54.5 \%$ ) and a healthy control (male, 50 years, BMI $24.161 .9 \mathrm{~kg} / \mathrm{m}^{2}$, no known diseases, no medication) were collected before and $60 \mathrm{~min}$ after mixed meal intake on EDTA $1.6 \mathrm{mg} / \mathrm{ml}$ to avoid coagulation and kept overnight at $4^{\circ} \mathrm{C}$ in the fridge. Density gradient centrifugation was performed in an initial step to separate microbial cells from blood cells. Thus, $5 \mathrm{ml}$ of $80 \%$ Nycodenz solution was added at the bottom of a tube containing $5 \mathrm{ml}$ of blood, followed by centrifugation at $13000 \mathrm{rpm}, 4^{\circ} \mathrm{C}$ for $2 \mathrm{~h}$. After density gradient centrifugation, individual layers consisting of Supernatant and a Nycodenz layer could be separated. Each layer was individually collected and subjected to serial filtration through two consecutive $3 \mu \mathrm{m}$ and one $0.22 \mu \mathrm{m}$ pore size polycarbonate filters (GTTP type, $0.2 \mu \mathrm{m}$ pore size PC membrane, $25 \mathrm{~mm}$ diameter, Merck Millipore, Germany). After filtration, all filters were washed in $1 \times$ UV-ed PBS buffer and were immersed in 4\% paraformaldehyde solution (PFA) in $1 \times$ UV-ed PBS buffer for $2 \mathrm{~h}$ at room temperature. Following chemical fixation, filters were washed in $1 \times$ UV-ed PBS buffer, dehydrated in $80 \%$ ethanol, air dried, and stored at $4^{\circ} \mathrm{C}$ for CARDFISH procedure.

CARD-FISH was performed following the standard protocol [54] with slight modifications. Permeabilization in Lysozyme solution (Sigma-Aldrich, MS, USA) $(10 \mu \mathrm{g}$ $\left.\mathrm{ml}^{-1}\right)$ in $0.1 \mathrm{M}$ Tris- $\mathrm{HCl}(\mathrm{pH}=7.8)$ and $0.05 \mathrm{M}$ EDTA $(\mathrm{pH}=8)$ buffer was done for $30 \mathrm{~min}$ at $37^{\circ} \mathrm{C}$ followed by $0.01 \mathrm{M} \mathrm{HCL}$ for $10 \mathrm{~min}$ at room temperature (RT) with washing steps of $1 \mathrm{~min}$ at RT in ultrapure water in between treatments. Hybridization of filter pieces belonging to both $3 \mu \mathrm{m}$ and $0.2 \mu \mathrm{m}$ filters took place for $3 \mathrm{~h}$ at $46^{\circ} \mathrm{C}$ in a pre-warmed hybridization buffer containing $0.9 \mathrm{M} \mathrm{NaCl}, 20 \mathrm{mM}$ Tris- $\mathrm{HCl}(\mathrm{pH}=7.5), 10 \%(\mathrm{w} / \mathrm{v})$ dextran sulfate, $0.02 \%(\mathrm{w} / \mathrm{v})$ SDS, $35 \%(\mathrm{v} / \mathrm{v})$ formamide 
(Fluka, Waltham, USA), and 1\% (w/v) blocking reagent (Boehringer, Mannheim, Germany). The HRP-labeled probe applied is specific for bacteria (EUB 338, [55]) and was used at a concentration of $0.166 \mathrm{ng} \mathrm{m}^{-1}$ (HRPprobe stock solution of $50 \mathrm{ng} \mathrm{ml}^{-1}$ diluted $1: 300 \mathrm{v} / \mathrm{v}$ in hybridization buffer). Following hybridization, filter pieces were incubated in $50 \mathrm{ml}$ of pre-warmed washing buffer containing $70 \mathrm{mM} \mathrm{NaCl}, 5 \mathrm{mM}$ EDTA ( $\mathrm{pH}=8.0$ ), $20 \mathrm{mM}$ Tris- $\mathrm{HCl}(\mathrm{pH}=7.5)$, and $0.01 \%$ SDS for $15 \mathrm{~min}$ at $48^{\circ} \mathrm{C}$. After washing, filters were incubated for $15 \mathrm{~min}$ at $\mathrm{RT}$ in $1 \times \mathrm{PBS}(\mathrm{pH}=7.6)$ to equilibrate the HRPlabeled probe. Subsequently, tyramide deposition was performed by incubating the filters for $20 \mathrm{~min}$ at $46^{\circ} \mathrm{C}$ in the dark in amplification buffer containing $1 \times$ PBS, 2 $\mathrm{M} \mathrm{NaCl}, 0.1 \%(\mathrm{w} / \mathrm{v})$ blocking reagent, $10 \%(\mathrm{w} / \mathrm{v})$ dextran sulfate, $0.0015 \%(\mathrm{v} / \mathrm{v}) \mathrm{H}_{2} \mathrm{O}_{2}$, and $1 \mu \mathrm{g} \mathrm{ml}^{-1}$ Alexa 594labeled tyramides (ThermoFisherScientific, MA, USA). Afterwards, the hybridized filters were rinsed in $1 \times$ PBS for $10 \mathrm{~min}$ at RT followed by staining with 4',6-diamidino-2-phenylindole (DAPI) $1 \mu \mathrm{g} \mathrm{ml}^{-1}$ for $10 \mathrm{~min}$ at RT, washing in ultrapure water, air dried, and embedded in mounting medium (a mixture of low fluorescence glycerol mountant (Citifluor AF1, London) and mounting fluid Vecta Shield (Vecta Laboratories, CA, USA)) in a $4: 1 \mathrm{v} / \mathrm{v}$ ratio and stored at $-20^{\circ} \mathrm{C}$ prior to imaging.

\section{Microscopic investigation}

Approximately 20-30 randomly selected fields of view (each of $15130.8 \mu \mathrm{m}^{2}$ ) were imaged on each hybridized filter piece from $3 \mu \mathrm{m}$ and $0.2 \mu \mathrm{m}$ pore size hybridized filters. The microscopic evaluation of the hybridized filters was done using a fluorescence microscope (Imager. Z2, Zeiss, Germany) with $\times 20$ air (numerical aperture $(\mathrm{NA})=0.5)$ and $\times 63$ oil $(\mathrm{NA}=1.4)$ objectives. On the $3 \mu \mathrm{m}$ filters, we observed predominantly unhybridized blood cells of different sizes for both Supernatant and Nycodenz filtrated samples, while hybridized bacterial cells were constantly found on the $0.22 \mu \mathrm{m}$ filters of the Nycodenz filtrated samples, occasionally in very low abundances also on the $0.22 \mu \mathrm{m}$ filters of the Supernatant filtrated samples (data not shown). Bacterial counts were performed only on the $0.22 \mu \mathrm{m}$ filters from the Nycodenz filtrate layer on 20 to 22 randomly selected fields of view.

\section{Results}

Our aim was to interrogate putative bacterial signatures related to obesity and characterize their potential dynamics after weight loss and metabolic alleviation following bariatric surgery (complete flowchart approach available under Additional file 1: Fig. S1-full description under the "Methods" section). For this, we started by investigating the predominant signature and taxonomy in a cross-sectional approach in blood samples from subjects at baseline (Table 1-baseline cohort characteristics of subjects with complete follow-up ( $\mathrm{n}=48$ ), Additional file 1: table $\mathrm{S} 1$-baseline cohort characteristics with initial matching $(\mathrm{n}=64))$ and went on to further explore the change in taxonomy in blood samples available for all subjects with follow-up (Tables 1 and 3) at months 3 and 12 after the procedure. To explore bacterial signatures related to weight loss and diabetes alleviation, similar analyses were done in good vs poor responders (Additional file 1: table S2-phenotypes of good vs poor responders, Additional file 1: Fig. S3).

\section{Contaminant-aware analyses allow the derivation of a core blood bacterial signature}

After filtering and removing contaminating ASVs (Additional file 2: Table S2), a distinct bacterial profile remained consisting of 20 phyla (Additional file 2: Table S4). Of the 2746 detected ASVs, 65 could not be assigned at the phylum level (2.4\%). Assigned taxonomy was dominated by Proteobacteria (59.0\%), Firmicutes (12.4\%), Patescibacteria (11.97\%), Cyanobacteria (8.9\%), and Actinobacteria (2.3\%), whereas $5.41 \%$ of ASVs belonged to various other phyla.

$21.6 \%$ of all ASVs (592) could not be assigned at the genus level. Assigned ASVs belonged to 314 genera and the most abundant genera included Aliterella, Anoxybacillus, Lactobacillus, and Sphingomonas from the three dominant phyla (Fig. 1A). The core bacterial signature consisting of 10 phyla and 120 genera with an overall abundance of more than $10 \%$ in the dataset could be recovered at each timepoint, although quantitative changes could be tracked over time and within disease and response groups (see further results) (Fig. 1B, C).

\section{Bacterial community composition is influenced by medication and reflects metabolic health}

Twenty-seven available host variables (Additional file 1: tables S4, S5) were fit onto genus-level RDA in all available samples with no missing data $(\mathrm{n}=101)$. RDA showed that $39.7 \%$ of the observed variance could be explained by the collected host variables $\left(R^{2}=0.3969, R^{2}\right.$ adj $=0.162$, $p$ values of Anova $=0.004)$. Associations with RDA ordination on Hellringer transformed abundances were most notable for timepoint (envfit; $\mathrm{R}^{2}=0.2184, \mathrm{p}=0.001$ ), white blood cell count (envfit; $\mathrm{R}^{2}=0.2531, \mathrm{p}=0.001$ ), BMI (envfit; $\mathrm{R}^{2}=0.2219, \mathrm{p}=0.001$ ), total fat mass (envfit; $\mathrm{R}^{2}=$ $0.2090, \mathrm{p}=0.001$ ), bacterial quantity (envfit; $\mathrm{R}^{2}=0.1788, \mathrm{p}$ $=0.001$ ), and triglycerides (envfit; $\mathrm{R}^{2}=0.1560, \mathrm{p}=0.001$ ). Several other significant associations with bacterial composition were observed for general host characteristics such as age, T2D status, MetS status, sex, anthropometric variables such as waist-to-hip ratio and visceral fat rating, and markers of metabolic 


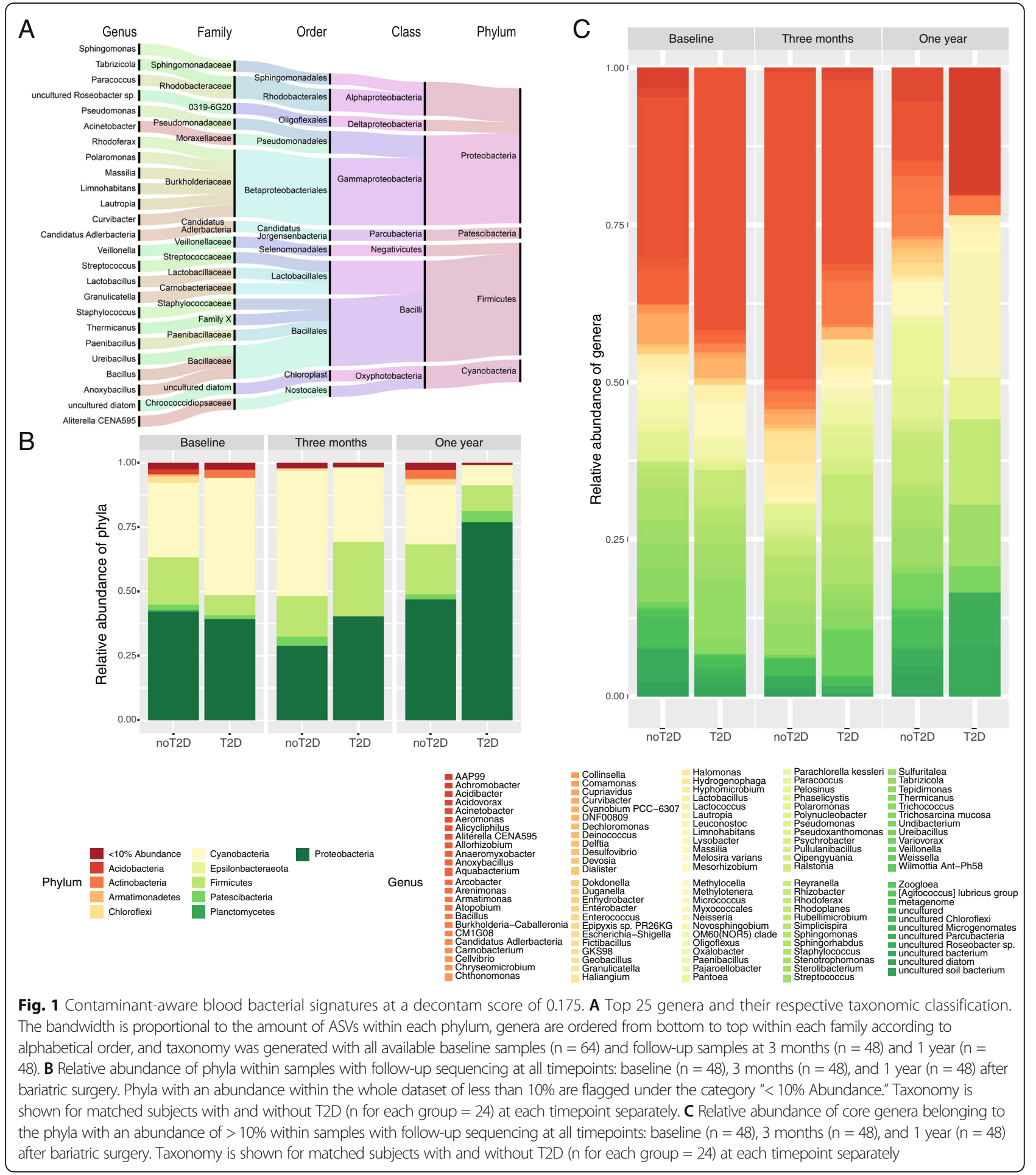

control including HDL cholesterol and HbA1c as well as inflammation markers like CRP and bacterial DNA burden (Additional file 1: table S4: envfit output for 27 variables on genera level RDA, Additional file 1: Fig. S4). Similar results were observed at ASV levels as well (Additional file 1: table S5).
Interestingly, while the number of antihypertensive, antidiabetic, and antihyperlipidemic medication was not correlated with ordination, the use of metformin and PPI significantly contributed to variance in composition (metformin $\mathrm{R}^{2}=0.0380, \mathrm{p}=0.022$; PPI $\mathrm{R}^{2}=0.0674, \mathrm{p}=$ 0.001; Additional file 1: tables S4, S5). 
Blood bacterial signature allows robust classification of subjects with metabolic syndrome

Cardiovascular disease and mortality are increased in subjects with MetS [56] even in the absence of T2D. Because MetS status was significantly associated with bacterial composition beyond single risk factors used in the MetS definition [27] (Additional file 1: tables S4, S5), we sought to investigate if bacterial composition could predict MetS status. For this, we applied random forest classification on the genus- as well as phylum-level abundances (20 phyla and 314 genera) to develop a classification index. The resulting MetS classification index (MetS- $\mathrm{I}_{\text {genus }}$ and MetS- $\mathrm{I}_{\text {phylum }}$ ) could robustly classify subjects along their actual clinical group at baseline and performed better at genus than at phylum level (AUCgenus $=0.816,95 \% \mathrm{CI}$ 0.661-0.9705 (DeLong) and AUCphylum $=0.740$ 95\% CI 0.589-0.890 (DeLong)) (Fig. 2A-D). MetS-I at both genus and phylum levels at baseline was correlated with several markers related to obesity, visceral fat distribution, insulin resistance, and inflammation from all timepoints, but was negatively associated with bacterial DNA load in the blood (Fig. 2E, F). The largest associations with host variables were seen for MetS- $\mathrm{I}_{\text {genus, }}$ which was positively correlated with anthropometric markers related to obesity and insulin resistance such as total fat mass, waist circumference, and WHR. It was also positively associated with inflammation reflected in white blood cell count, CRP, and LBP as well as metabolic markers related to obesity and insulin resistance such as HOMA-IR, HbA1c, FPG, FPI, and uric acid. The MetS-I $\mathrm{I}_{\text {genus }}$ was further related to dyslipidemia and hypertension with positive correlations noted for triglyceride levels and number of antihypertensive drugs, while being negatively associated with HDL cholesterol. Moreover, both MetS- $\mathrm{I}_{\text {genus }}$ and MetS- $\mathrm{I}_{\text {phylum }}$ were negatively associated with bacterial quantity (Fig. 2E, F, Table 2).

T2D is characterized by a loss of health-associated genera and a decreased ASV richness

We investigated bacterial alpha diversity according to T2D status: At baseline, subjects with T2D ( $\mathrm{n}=24)$ displayed significantly lower observed richness as compared to subjects without T2D $(\mathrm{n}=24)$ (mean observed richness $=28 \pm 2.31$ vs $19 \pm 2.10, \mathrm{p}=0.039$ in subjects without T2D and with T2D respectively; Fig. 3A). T2D was associated with significant shifts in several genera as compared to subjects without T2D: At baseline, significantly lower abundances of Anoxybacillus, Duganella, Acidibacter, and Chryseomicrobium as well as Sphingomonas are found in T2D (Fig. 3B). Furthermore, timepoint seemed to be relevant for the differences in bacterial abundances between subjects with and with T2D (Additional file 1: Fig. S5A, B). There were no significantly differentially abundant ASVs between T2D and nonT2D overlapping between baseline and 1 year, but the differences between subjects with and without T2D seemed to be shifting gradually. While subjects with T2D at baseline and 3 months showed a reduced abundance in Sphingomonas, a common feature seen between T2D at 3 months and 1 year was reduced Pseudomonas in T2D. Congruent T2D-associated features seen at baseline and 1 year were reduced Bacillaceae and Burkholderiaceae (Additional file 2: Table S7). Some of the reduced genera in T2D were expectedly negatively associated with markers of metabolic disease and inflammation such as Anoxybacillus and Sphingomonas at baseline with leukocytes and blood pressure respectively (Fig. 3C) as well as Paracoccus with HOMA-IR and leukocytes and Rhodoferax with markers of obesity at 1 year post-bariatric surgery (Additional file 1: Fig. S5C). Other genera were surprisingly positively associated with BMI such as Sphingomonas (at months 3 and 12) or with lipids and blood pressure such as Acidibacter (at baseline, months 3 and 12) (Fig. 3C, Additional file 1: Fig. $\mathrm{S} 5 \mathrm{C})$

\section{Differential response in weight loss and glucose metabolism following bariatric surgery}

As expected, bariatric surgery had a marked impact on BMI, body composition (WHR, total fat mass, and visceral fat ratio), metabolism, and inflammation as early as 3 months post-procedure (Table 3). From 24 subjects with T2D at baseline, nine subjects were still classified as T2D after 3 months, and only four after 1 year. From patients without T2D at 1 year, eight had impaired fasting glucose (prediabetes) and classified therefore as subjects without T2D alleviation after bariatric surgery. From the total 48 subjects with complete follow-up data, 24 subjects were categorized as good responders (i.e., losing more than $60 \%$ of excess BMI within 1 year), whereas 13 subjects were classified as poor responders (Additional file 1: table S2, Fig. S3). Poor responders not only lost less weight, but also benefited less from bariatric surgery in regard to improvement of lipid metabolism, insulin resistance, or inflammation, as they still displayed significantly higher HOMA-IR, triglycerides, LDL cholesterol, and CRP compared to good responders 1 year after bariatric surgery (Additional file 1: table S2).

\section{Reduced bacterial blood load in T2D is ameliorated after bariatric surgery}

Bacterial DNA could be measured in 127 blood samples out of 144 samples with available follow-up data at all three timepoints at a quantity ranging from 4.63 to $140.4 \mathrm{fg}$ in $200 \mu \mathrm{l}$ blood (median $=21.6 \mathrm{fg}$ ). Albeit using the same procedure and the same kit, total extracted DNA varied widely between samples spanning from 12.7 


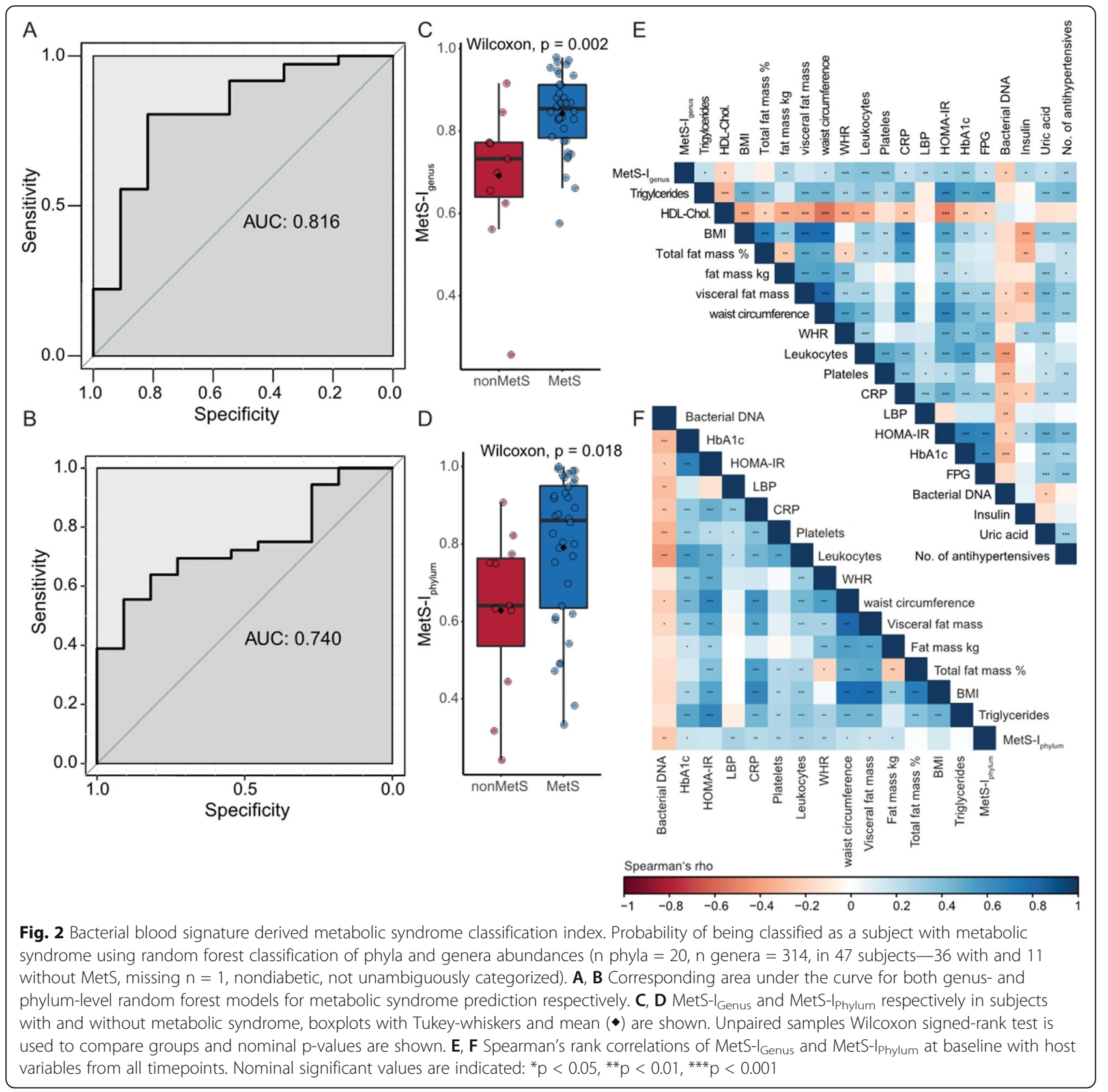

to $98.2 \mathrm{ng}$ total $\mathrm{DNA} / \mu \mathrm{l}$ blood leading to a corrected range of bacterial DNA quantity within $0.08-4.11 \mathrm{pg}$ per $\mu \mathrm{g}$ total DNA (median $=0.7 \mathrm{pg}$ bacterial DNA per $\mu \mathrm{g}$ total DNA). While bacterial DNA could be detected in negative controls at $0.162 \mathrm{pg}$ per total $\mu \mathrm{g}$ DNA, total DNA in negative controls did not exceed $1.2 \mathrm{ng} / \mu \mathrm{l}$ leading to total bacterial DNA amounts of less than $1.5 \mathrm{fg}$ $($ median $=0.4 \mathrm{fg})$. Overall, bacterial blood load was positively associated with bacterial richness at baseline (Fig. 4A) and negatively with leukocytes (Fig. 4B). This observation was further validated in a replication cohort consisting of 62 subjects from the same center (Additional file 1: table S3). Correlation analyses in the replication cohort further underline the negative associations between inflammation and bacterial load and the association between LBP and inflammation as well as obesity (Additional file 1: table S6).

Spanning over all timepoints, bacterial load was negatively correlated with waist circumference, $\mathrm{HbAlc}$, and inflammation markers such as leukocytes as well as markers related to a presumed leaky gut such as LBP (Additional file 1: Fig S6A-D, tables S7, S8). The latter was on the other 
Table 2 Spearman correlations between MetS-I genus at baseline with host variables ( $n=141,47$ subjects at 3 timepoints)

\begin{tabular}{lll}
\hline Variables & Rho & p-value \\
\hline CRP & 0.21 & $<0.05$ \\
Fasting glucose & 0.2 & $<0.05$ \\
Triglycerides & 0.2 & $<0.05$ \\
HDL cholesterol & -0.19 & $<0.05$ \\
HbA1C & 0.33 & $<0.0001$ \\
Insulin & 0.27 & $<0.5$ \\
Total fat mass & 0.26 & $<0.01$ \\
Number of antihypertensive drugs & 0.27 & $<0.01$ \\
Platelet count & 0.38 & $<0.0001$ \\
Serum albumin & 0.18 & $<0.05$ \\
Uric acid & 0.2 & $<0.05$ \\
White blood cell count & 0.38 & $<0.0001$ \\
LBP & 0.25 & $<0.001$ \\
Bacterial quantity & -0.23 & $<0.05$ \\
\hline
\end{tabular}

hand associated with markers of metabolic disease and obesity (Additional file 1: table S7). While these correlations were weak to moderate, the use of Spearman's rank correlation made them less likely to be influenced by a few high leverage points as shown after removing statistical outliers for bacterial quantity (Additional file 1: Fig. S7A-D). Accordingly, subjects with T2D at baseline displayed lower bacterial quantity in both our study and replication cohorts (Fig. 4C).

Blood bacterial load was decreased 3 months postbariatric surgery (median bacterial quantity in $\mathrm{pg} / \mu \mathrm{g}$ at baseline $=0.032$, vs 0.024 at 3 months, $\mathrm{p}=0.013$, Fig. 4D), but increased significantly at 1 year following surgery (median bacterial quantity in $\mathrm{pg} / \mu \mathrm{g}$ at 1 year = 0.062 , p-value compared to 3 months $=1.1 \times 10^{-6}, \mathrm{p}$ value compared to baseline $=0.002$, Fig. $4 \mathrm{D})$ independently of T2D status at baseline (Additional file 1: Fig S8A, B). When checking the changes according to weight loss response, subjects deemed "good responders" and "poor responders" displayed similar blood bacterial load at baseline (median bacterial quantity in $\mathrm{pg} / \mu \mathrm{g}$ "good responder" $=0.864$ vs "poor responder" $=0.866$, $\mathrm{p}$-value $=0.9)$ but blood bacterial quantity at 1 year increased less dramatically in good responders and was not significant as compared to the increase in blood bacterial quantity in poor responders (fold change 1.6 vs 2.1 in good vs poor responders respectively, Fig. 4E). Bacterial diversity, on the other hand, showed a transient significant decrease at 3 months only to increase again almost to baseline at 1 year in poor responders, while continuously and significantly decreasing in good responders (Fig. 4F).
LBP, expected to reflect host's response to bacterial DNA, decreased continuously and significantly after bariatric surgery (median LBP in $\mu \mathrm{g} / \mu \mathrm{l}$ at baseline $=20.47$ vs 15.99 at 3 months and 6.0 at 1 year after bariatric surgery, $\mathrm{p}_{3 \text { MonthvsBaseline }}=3.3 \mathrm{e}^{-5}, \mathrm{p}_{1 \text { Yearvs3Months }}=2.46 \mathrm{e}^{-6}$, $\mathrm{p}_{1 \text { YearvsBaseline }}=5.8 \mathrm{e}^{-9}$ ) independently of weight loss response or diabetes status at baseline (Additional file 1: Fig. S9A, B). Similarly, leukocytes decreased overtime after bariatric surgery, but less so and non-significantly in subjects with poor response (good responders: $\mathrm{p}_{3 \text { MonthvsBaseline }}=3.3 \mathrm{e}^{-5}, \mathrm{p}_{1 \text { Yearvs3Months }}=0.14$, and $\mathrm{p}_{1 \text { YearvsBaseline }}=6.5 \mathrm{e}^{-4}$ respectively; poor responders: $\mathrm{p}_{3 \text { MonthvsBaseline }}=0.67, \mathrm{p}_{1 \text { Yearvs3Months }}=0.08$, and $\left.\mathrm{p}_{1 \text { YearvsBaseline }}=0.16\right)($ Additional file 1: Fig. S9C).

\section{Metabolic improvement after bariatric surgery is accompanied by early changes in microbial differential abundances in subjects with and without T2D}

Beyond changes in blood bacterial load over time, there were significant and consistent shifts in bacterial genera over the whole cohort between months 3 and 12 after bariatric surgery as compared to baseline. Decreased ASVs belonged to genera such as Anoxybacillus, Rhizobacter, and Sphingomonas, whereas other genera such as Acinetobacter, Granulicatella, and Pseudomonas increased. Comparing shifts at 1 year and baseline showed that most of those seen at 3 months post-bariatric surgery were preserved at 1 year (Fig. 5A).

Moreover, T2D remission following bariatric surgery was associated with significantly lower proportions of Burkholderiacae, Veilonellaceae, and Lactobacillaceae and higher proportions of Rhodobacteraceae and Pseudomonales at all timepoints combined (Fig. 5B). Genera with observed shifts after bariatric surgery were closely tied to metabolic control: Genera, which had a lower abundance after surgery such as Anoxybacillus, Rhizobacter, and Sphingomonas for example, were significantly positively associated with markers of body composition such as overall and visceral fat mass, BMI, and CRP. Furthermore, significantly positive correlations were observed for aforementioned genera with markers of metabolic control including HOMA-IR, number of antidiabetics, uric acid, triglycerides, and LDL cholesterol (Fig. 5D). As for genera being more abundant after surgery, CM1G08 and Acinetobacter were negatively associated with markers of central obesity and metabolic disease as well as inflammation, while being positively associated with HDL cholesterol and blood bacterial quantity. Contrarily, Granulicatella was negatively associated with excess BMI loss, but positively with sCD14. Of note, while Granulicatella was higher at 1 year compared to baseline, it seemed to increase at 3 months, while the increase in GM1G08 and Acinetobacter was continuous (Fig. 5A). Paracoccus-which was shown to 


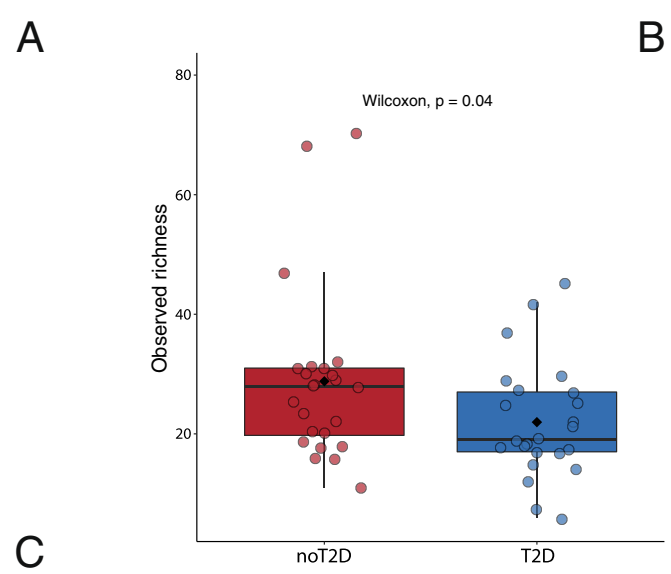

B Differentially abundant genera in T2D vs nonT2D

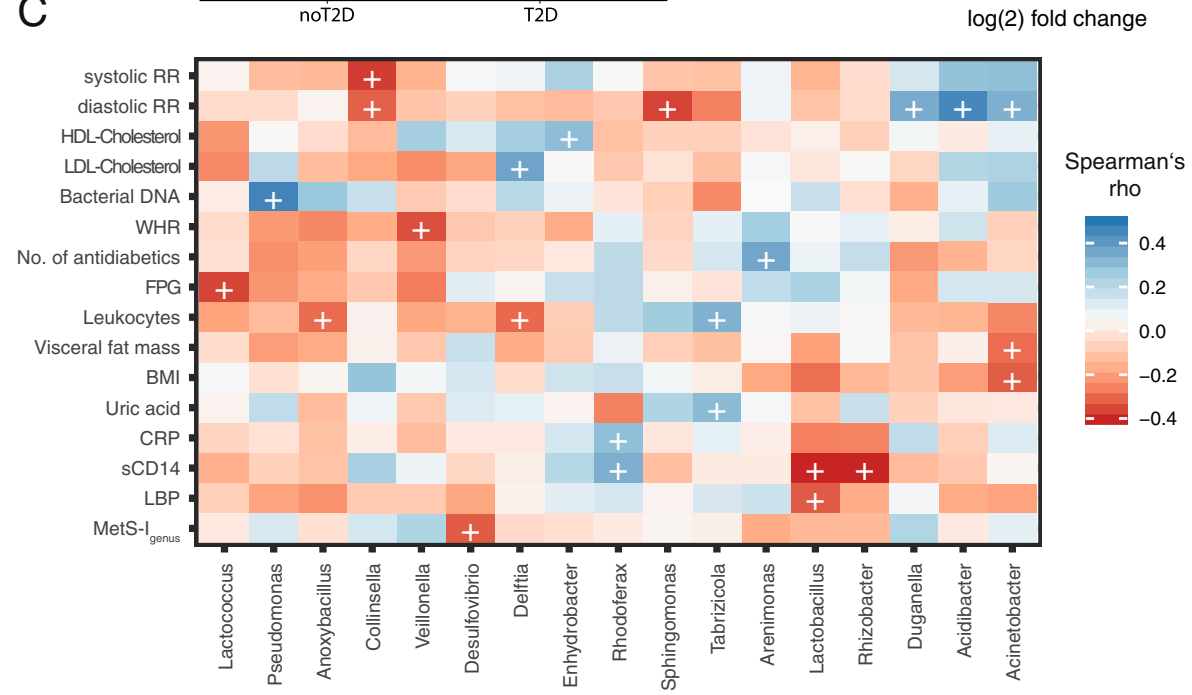

Fig. 3 A Observed richness between subjects with $(n=24)$ and without T2D $(n=24)$, boxplots with Tukey-whiskers and mean $(\bullet)$ as well as median are shown. Unpaired samples Wilcoxon signed-rank test is used to compare groups and nominal $p$-value is shown. B Differentially abundant genera in subjects with T2D $(n=24)$ compared to subjects without T2D $(n=24)$ at baseline. Differential abundance is calculated at ASV level using taxonomy after stringent control for contamination at a decontam score of 0.5 and reported at the genus level. C Spearman's rank correlations of relative selected genera abundance with host parameters at baseline. Selection included genera seen to be differentially abundant between groups (i.e., T2D, good/poor responders, and T2D alleviation vs no T2D alleviation). Only genera are shown with at least one significant correlation with host markers. (+) refers to $p$-value $<0.05$. Color represents correlation strength (Rho) according to color legend. Abbreviations: HDL high-density lipoprotein, LDL low-density lipoprotein, WHR waist to hip ratio, BMI body mass index, CRP C-reactive protein, sCD14 soluble cluster of differentiation 14, LBP lipopolysaccharide binding protein, EBL excess BMI loss

be decreased in T2D at baseline and increased in subjects experiencing T2D remission- was negatively associated with inflammation, FPG, triglycerides, HOMA-IR, and LBP (Fig. 5D).

Good responders displayed an increase in ASVs belonging to Streptococcaceae such as Lactococcus and streptococcus species, Burkholderiales such as Polaromonas and Polynucleobacter, Rhodobacteraceae such as Paracoccus, and Bacillaceae such as Anoxybacillus at all timepoints (Fig. 5C). While Paracoccus was associated with both decreased inflammation and metabolic impairment, some genera increased in good vs poor response were associated with increased BMI and inflammation such as Anoxybacillus or Thermicanus overall, but significantly decreased at 1 year when compared to baseline
(Fig. 5A). Several genera abundantly observed in good responders and subjects who experienced T2D remission, were initially decreased in T2D, further delineating a putative positive association with good metabolic health (Figs. 3B and 5B-D).

\section{CARD-FISH allows the visualization of living bacteria in the blood post-bariatric surgery}

In order to verify whether increased bacterial quantity post-bariatric surgery is only related to uptake of bacterial DNA or bacteremia, we sought to visualize bacterial cells in a subject, who had undergone bariatric surgery, in whom blood bacterial quantity could be measured at baseline and for whom bacterial sequence data are available. CARD-FISH implementation was unsuccessful in 
Table 3 Clinical and biological characteristics of study cohort subjects before, 3, and 12 months after the bariatric surgery procedure

\begin{tabular}{|c|c|c|c|}
\hline & $\begin{array}{l}\text { Baseline } \\
N=48\end{array}$ & $\begin{array}{l}\text { Three months post-bariatric } \\
\text { surgery } \\
N=48\end{array}$ & $\begin{array}{l}\text { One year post-bariatric } \\
\text { surgery } \\
N=48\end{array}$ \\
\hline \multicolumn{4}{|l|}{ General and body composition } \\
\hline Sex $(F / M)$ & & $32 / 12$ & \\
\hline Metabolic syndrome status, N (\%) & $36 / 47(76.6)^{\mathrm{A}}$ & $17 / 41(41.5)^{\mathrm{B}}$ & $10 / 43(23.3)^{C}$ \\
\hline Diabetes status, N (\%) & $24(50.0)^{\mathrm{A}}$ & $9(18.7)^{B}$ & $4(8.3)^{C}$ \\
\hline BMI $\left(\mathrm{kg} / \mathrm{m}^{2}\right)$ & $49.7 \pm 6.50^{A}$ & $41.3 \pm 5.85^{B}$ & $35.1 \pm 5.62^{c}$ \\
\hline WHR & $0.95[0.91 ; 1.02]^{\mathrm{A}}$ & $0.94[0.88 ; 0.99]^{\mathrm{A}}$ & $0.94[0.87 ; 1.01]^{\mathrm{A}}$ \\
\hline Total fat mass (in \%) & $49.4[43.3 ; 53.0]^{\mathrm{A}}$ & $46.0[38.8 ; 48.2]^{B}$ & $37.0[28.9 ; 44.0]^{C}$ \\
\hline Visceral fat mass ratio & $19.0[17.0 ; 22.0]^{\mathrm{A}}$ & $15.0[12.8 ; 16.0]^{B}$ & $12.0[8.00 ; 13.0]^{C}$ \\
\hline \multicolumn{4}{|l|}{ Glycemia, insulin resistance, and antidiabetic medication intake } \\
\hline HbA1c (\%) & $5.62[5.42 ; 6.66]^{\mathrm{A}}$ & $5.42[5.17 ; 5.77]^{\mathrm{B}}$ & $5.21[4.89 ; 5.64]^{\mathrm{C}}$ \\
\hline FPG $(\mathrm{mmol} / \mathrm{l})$ & $5.50[5.21 ; 7.54]^{\mathrm{A}}$ & $5.25[4.89 ; 6.04]^{\mathrm{B}}$ & $5.00[4.68 ; 5.57]^{C}$ \\
\hline HOMA-IR & $5.37[3.16 ; 8.85]^{\mathrm{A}}$ & $2.62[2.12 ; 2.93]^{B}$ & $2.30[1.07 ; 3.15]^{\mathrm{B}}$ \\
\hline \multicolumn{4}{|l|}{ Hypertension status } \\
\hline Systolic blood pressure (mmHg) & $132 \pm 12.9^{\mathrm{A}}$ & $120 \pm 14.9^{B}$ & $120 \pm 14.7^{B}$ \\
\hline Diastolic blood pressure (mmHg) & $76.6 \pm 12.1^{\mathrm{A}}$ & $68.2 \pm 13.0^{\mathrm{A}}$ & $68.8 \pm 11.4^{\mathrm{A}}$ \\
\hline \multicolumn{4}{|l|}{ Dyslipidemia and antihyperlipidemic medication intake } \\
\hline LDL-C (mmol/l) & $3.16[2.48 ; 3.81]^{\mathrm{A}}$ & $2.26[1.92 ; 2.83]^{\mathrm{B}}$ & $2.37[1.93 ; 2.91]^{\mathrm{B}}$ \\
\hline HDL-C (mmol/l) & $1.21[0.99 ; 1.40]^{\mathrm{A}}$ & $1.12[0.96 ; 1.35]^{\mathrm{A}}$ & $1.52[1.27 ; 1.59]^{\mathrm{B}}$ \\
\hline TG $(\mathrm{mmol} / \mathrm{l})$ & $1.68[1.28 ; 2.15]^{\mathrm{A}}$ & $1.15[0.94 ; 1.46]^{\mathrm{B}}$ & $0.96[0.64 ; 1.30]^{C}$ \\
\hline \multicolumn{4}{|l|}{ Blood and inflammatory markers } \\
\hline Leukocytes (Gpt/l) & $7.50[6.70 ; 8.50]^{\mathrm{A}}$ & $6.60[5.50 ; 8.03]^{\mathrm{A}}$ & $6.10[5.20 ; 7.32]^{\mathrm{A}}$ \\
\hline LBP $(\mu \mathrm{g} / \mathrm{ml})$ & $20.465[17.89 ; 24.21]^{\mathrm{A}}$ & $15.99[11.75 ; 17.59]^{B}$ & $6.005[2.36 ; 14.01]^{\mathrm{C}}$ \\
\hline sCD14 (ng/ml) & $3089 \pm 384^{\mathrm{A}}$ & $3626 \pm 323^{B}$ & $3243 \pm 385^{c}$ \\
\hline CRP $(p g / m l)$ & $8.61[3.87 ; 18.8]^{\mathrm{A}}$ & $4.56[1.49 ; 10.1]^{\mathrm{B}}$ & $1.24[0.43 ; 3.52]^{C}$ \\
\hline Bacterial DNA amount (in pg per $\mu$ g extracted whole DNA) & $0.64[0.48 ; 1.05]^{\mathrm{A}}$ & $0.48[0.32 ; 0.68]^{B}$ & $1.24[0.90 ; 2.03]^{C}$ \\
\hline
\end{tabular}

Median and first, as well as third, quartile limits (median [q1; q3]) are shown for non-normally distributed, continuous variables. For normally distributed, continuous variables, data are given in mean \pm standard deviation (mean \pm SD). For categorical parameters, total numbers (percentage) are shown. Significant $\mathrm{p}$ values are depicted according to group differences: the difference between 2 values in a row with the same letter is non-significant. Abbreviations: BMI body mass index, CRP C-reactive protein, FPG fasting plasma glucose, HbA1c glycated hemoglobin A1c, HDL high-density lipoprotein, HOMA-IR homeostatic model assessment for insulin resistance, $L B P$ lipopolysaccharide binding protein, $L D L$ low-density lipoprotein, $s C D 14$ soluble CD14, TG triglycerides, WHR waist to hip ratio

frozen blood samples, but considering blood bacterial quantity increased after surgery over time, we hypothesized that this state is more likely to yield bacterial cell visualization. Because bacteremia and endotoxemia have been associated with chewing and food intake $[5,57,58]$, blood samples were also collected $1 \mathrm{~h}$ after a mixed meal to increase chances of visualization. Similarly, samples were taken from a healthy athletic control, whose weight has been stable for the last 2 years and who has no known diseases and no medication.

Our CARD-FISH results show the presence of intact bacterial cells in the blood samples from the patient collected before and after mixed meal intake with an abundance of $7.9 \times 10^{4}$ cells ml ${ }^{-1}$ and $1.2 \times 10^{5}$ cell ml $^{-1}$ respectively (Additional file 1: Fig. S10A, B, Additional file 2: Table S8) in support of increased bacteremia after food intake and chewing. In contrast, blood samples from the healthy control revealed no presence of hybridized bacteria, although filter pieces from all filters $(3 \mu \mathrm{m}$ and $0.2 \mu \mathrm{m})$ used for cell separation of both the Supernatant and Nycodenz layers were hybridized and imaged (Additional file 1: Fig. S10 C,D). An additional control to certify the CARD-FISH was successful and the lack of hybridized cells in the healthy subject is not due to a technical error, the same control blood sample was deliberately contaminated with Pseudomonas putida (DSM6125) (Additional file 1: Fig. S10 E,F). Therein, positively hybridized $P$. putida cells and no other cell morphotypes could be observed, adding evidence that freshly collected blood samples from the healthy control do not contain bacteria at abundances that can be detected by CARD-FISH. 


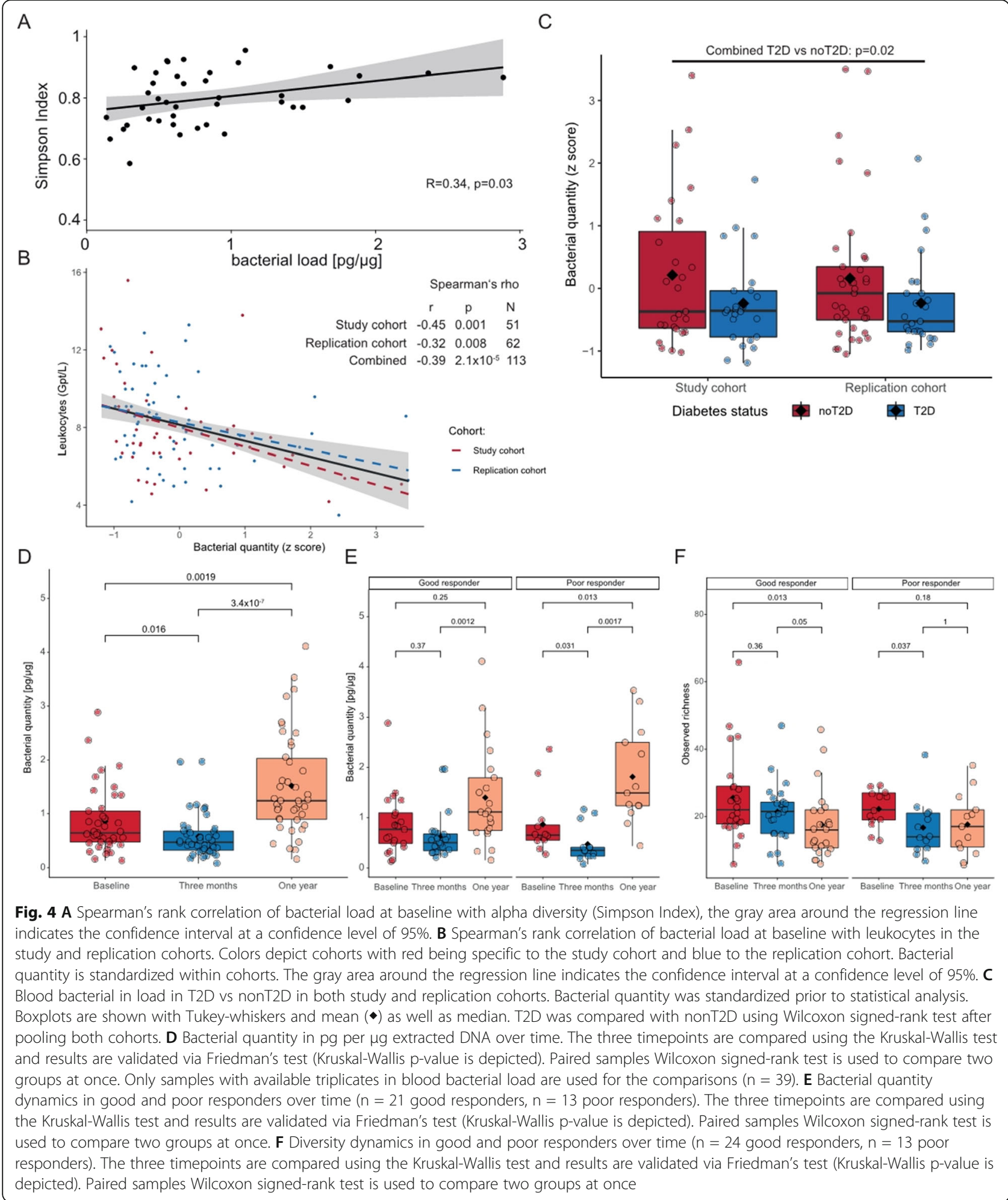

\section{Discussion}

The presence of bacteria and bacterial products in various tissues and their contributions to the local and systemic inflammation have been suggested as novel mechanisms for both development and progression of "non-communicable" metabolic diseases, such as obesity [13-15, 17, 19, 22], T2D [13-15], cardiovascular diseases [17], and cirrhosis [18, 59]. However, studies supporting this hypothesis are mostly limited by the lack of control for contamination and a high overestimation of bacterial 


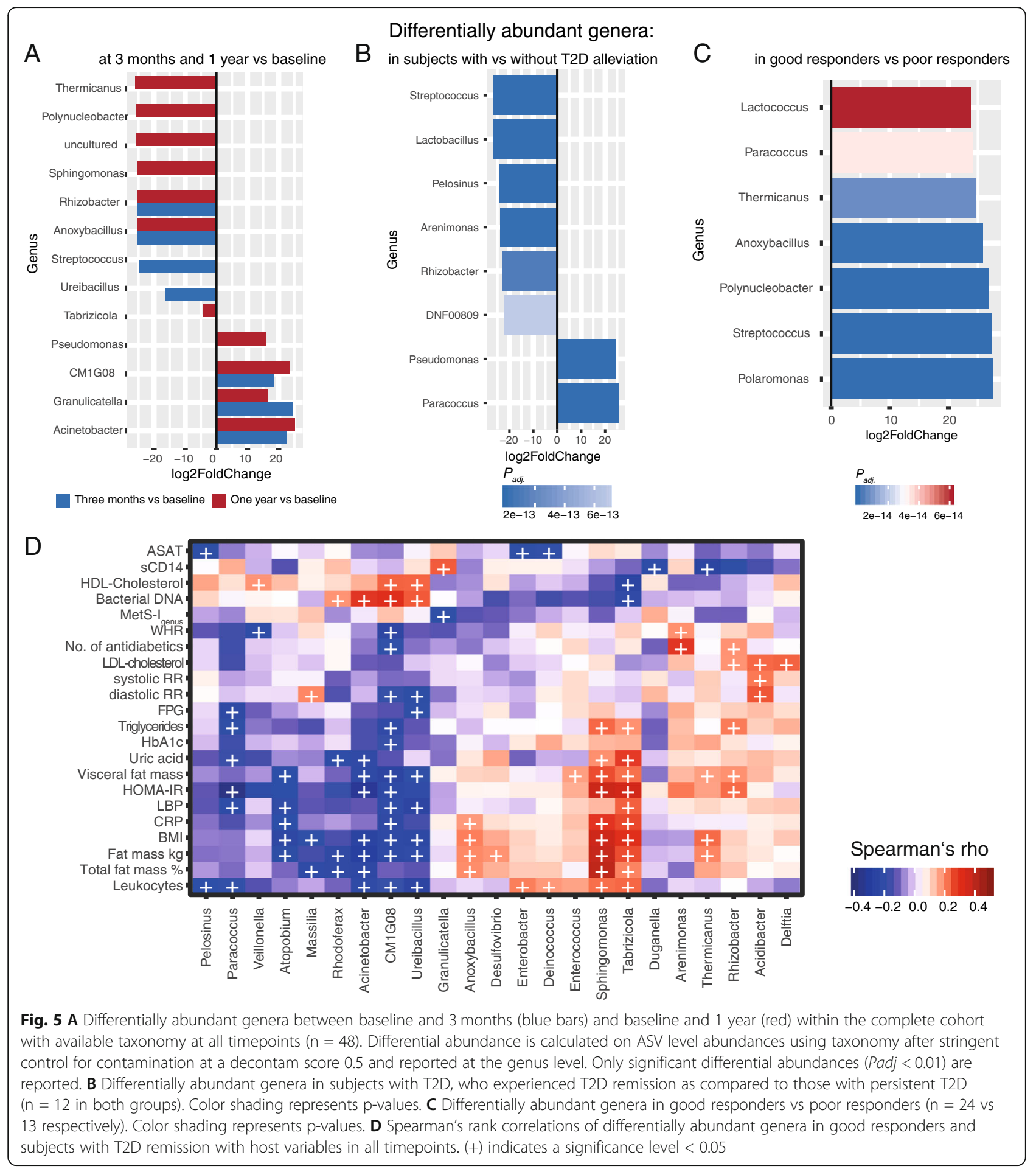

DNA quantity, consequently inflating results. In the present work, we therefore quantified and characterized bacterial DNA in the blood of subjects with high metabolic burden undergoing bariatric surgery with an emphasis on including suitable negative controls and subsequently substracting identified contaminant in downstream analyses. The goal was to identify links between metabolism and a putative blood bacterial signature as well as its dynamics over time after bariatric surgery.

After stringent experimental and bioinformatic control for contaminants, we report 2746 features (ASVs) belonging mostly to the phyla Proteobacteria and Firmicutes, which corroborates previously published results 
$[13,14,21,59,60]$. Furthermore, we evidence quantitative, compositional, and taxonomic signatures associated with markers of metabolic disease and T2D and shifts thereof driven by medication and weight loss intervention. We also provide qualitative and quantitative evidence for the presence of bacterial cells in the blood samples of a subject with obesity, who has undergone bariatric surgery as well as bacteremia after mixed meal intake in comparison to a healthy control.

Our results provide support for the existence of an individually determined core circulatory bacterial signature and microbiome, which reflects disease and intervention with $40 \%$ of observed variance in bacterial composition explained by 27 host variables prominently related to inflammation, anthropometric measures, and metabolic health and medication intake. Of the latter, notable associations of bacterial signature were seen for metformin and PPI intake. These results underscore findings from other studies relying on the application of multi-method characterizations including evidence of viability to debunk the notion that the blood is free of viable microorganisms $[14,59-64]$ as well as studies showing the deeply underrated modulatory effect of medication on host microbial communities and the importance of assessing drug intake in microbial surveys of any niche [65].

Moreover, blood bacteria signature allowed the classification of subjects with and without MetS along their actual clinical group and more accurately so at genus than phylum level. This is in line with recent evidence showing disease-specific tissue signatures in several tissues pertaining to metabolic health as well as cancer $[13,14,21]$ and could be related to the particular vulnerability to translocation of specific ingested bacteria in respective diseases. Together, these independent observations reinforce the notion of a predictive circulatory metabolic bacterial signature reminiscent of the described enterotypes [66] of the gut microbiome.

Similarly, we show a specific bacterial signature for T2D characterized by reduced bacterial diversity and an overall reduction in genera belonging to Firmicutes and Proteobacteria. Diversity scores were negatively correlated with inflammation, visceral adiposity, and uremia, echoing observations of diversity in gut microbial communities in T2D [67]. Although the reported reduction in genera is in line with data from Anhê et al., we did not evidence their reported reduction in Bacteroidetes nor the increase in E. coli in T2D [13]. While we evidence ASVs belonging to Bacteroidetes, their prevalence was very low and was not particularly associated with metabolic control in our cohort. This could be indeed due either to cohort-specific characteristics or our choice in primers. Our data are more in accordance with results from another cohort based in our center. On the other hand, we report several more genera in the blood
(314 with 120 core genera), which possibly reflects our choice of whole blood as material compared to plasma samples used by Anhê et al.

A hallmark for T2D in our study was the loss of genera related to Bacillaceae and Bukholderiaceae. Several of the reduced genera were associated with a healthier metabolic status. Others, such as Sphingomonas and Acidibacter, were positively associated with BMI and insulin resistance as well as increased blood pressure and antihyperlipidemic medication respectively. Considering subjects with and without T2D at baseline were matched on BMI and did not differ significantly in blood pressure nor lipid levels but were significantly more medicated, these differences could arise from medication effects. This is supported by evidence from Anhê et al. showing increased Sphingomonas in subjects with T2D (at least in adipose tissue) and by the fact that these differences disappear after bariatric surgery in our cohort over time, when subjects are taken off their antihyperlipidemic and antihypertensive medication sequentially.

Bariatric surgery, on the other hand, led to swift changes in metabolism and bacterial composition in the blood: Changes occurring after bariatric surgery take place early on and are mostly stable over time, which is in line with available data on the rearrangement of the gut microbiome after bariatric surgery [68]. In addition, subjects who experienced T2D remission showed a significant decrease in several clades associated with cardiometabolic and cardiovascular disease such as Streptococcaceae [69], Veilonellaceae [70], or Lactobacillales [71] which are evidenced to be increased in T2D and under diabetic medication in the gut. In contrast, a few health-related genera increased after bariatric surgery, i.e., genera associated with improved insulin sensitivity and ameliorated lipid status or reduced adiposity at baseline in our cohort. Similarly, subjects who experience T2D remission after bariatric surgery displayed increases in genera seen to be initially decreased in T2D, further delineating a positive putative association of these bacteria with metabolic health.

We further investigated circulating bacterial DNA load: Quantification of 16s rRNA showed that bacterial load was positively associated with bacterial diversity and that the increase of the very small bacterial quantity observed after bariatric surgery reflects a reduced inflammatory tone and an improved metabolic health, which we found intriguing. This was also supported by the observations that (a) bacterial load is reduced in T2D at baseline, also in our independently measured replication cohort; (b) bacterial load was negatively associated with metabolic disease index score at baseline; (c) bacterial load was associated with bacterial composition in the opposite direction dictated by metabolic risk factors such as HbA1c, BMI, and TG; and (d) it increased 
after bariatric surgery. This is further corroborated by the fact that all associations with bacterial quantity are positive with health markers at each and all timepoints. There was no significant difference in bacterial load at baseline between good and poor responders but poor responders experienced an even more pronounced increase in bacterial load. Unexpectedly, bacterial diversity continuously and significantly decreased in good responders, while in poor responders it significantly dipped around 3 months only to almost recover at 1 year post-bariatric surgery. This was paralleled by similar dynamics for leukocytes in good responders, while poor responders failed to display a significant decrease in leukocytes and inflammation over time. Considering bacterial diversity was still negatively associated with leukocytes at all timepoints, it seems likely that the superior increase in bacterial quantity in poor responders is the driving factor behind the observed increased diversity. The latter, on the other hand and along increased bacterial quantity, could be at least partially responsible for the sustained inflammation seen at 1 year in poor responders. Thus, the increased bacterial quantity after bariatric surgery could be related to increases in gut permeability [72] and transmissibility of oral bacteria after the surgery due to increased $\mathrm{pH}$ [73]. Stronger increases thereof in poor responders are possibly related to an even more impaired gut permeability due to sustained obesity [74] as well as increased food intake. This begs the question of the transient reduction in bacterial quantity at 3 months post-bariatric surgery: Considering the origins of bacterial DNA we see are very likely environmental (i.e., food and oral bacteria), bacterial quantity reduction at 3 months could be related to the extreme restriction of food intake, far more pronounced at 3 months than around 1 year after surgery where eating behavior and weight loss are more stable. In support of this, bacterial diversity decreases at 3 months in both good and poor responders only to increase in poor responders again, which could indeed be due to increased food intake. It is important to note that the observations are based on an increase in miniscule amounts of bacterial DNA far from the expected quantity in clinically relevant bacteremia and sepsis.

We would like to acknowledge limitations in our study: Although we control for contamination starting at DNA isolation including filling EDTA vials with PBS via needle butterflies to take production line contamination of medical products into account, we cannot fully exclude contamination via puncture of the skin or other environmental sources. Of note, the highest diligence was used in skin decontamination prior to venipuncture and DNA isolation vials were collected as the last vials (in a sequence of 12 vials). Moreover, we did not observe typical skin bacteria among identified contaminants, making the skin as a source of contamination unlikely.
Spurious environmental contamination cannot be fully excluded and might explain the encounter of Choloroflexi, Rhizobacter, Limnohabitans, and Plactomycetes as well as uncultured diatom in our dataset. While we aimed to reduce these spurious findings as much as possible in a data-driven manner to avoid arbitrary preselection of taxa, even complete subtraction of contaminant AVSs selected via stringent decontam score as done by Poore et al. [21] did not eliminate these taxa from the dataset completely. This commends the development of even better pipelines and bioinformatics tools for decontamination in small bacterial biomass samples. An encouraging observation, nonetheless, is that these taxa do not seem to be particularly relevant for our observed phenotype associations nor does a more stringent decontamination score change the conclusion of our work, further supporting the emergence of disease signatures in circulating bacterial composition.

We evidence circulatory bacterial DNA but can only speculate about its origins: The translocation of bacteria from the gut has been the primary considered mechanism. Although this hypothesis is tempting, we did not validate it by performing gold standard gut permeability tests. Moreover, evidence from the HMP have shown that the blood bacterial signature closely resembles that from the skin and oral cavity [61], further pointing to alternative or additive origins. Considering transmission of oral strains along the gastrointestinal tract is more common than previously recognized [75-77] and that gut rearrangement after bariatric surgery is associated with increased $\mathrm{pH}$ in the gut, a shift in bile acid pools [73], and increased PPI prescription especially after RYGB also evident in our cohort [78], we cannot exclude that the change in the circulatory blood bacterial signature is reflective of changes in these, among several other bacterial host niches. The increase in blood bacterial load after bariatric surgery could be seen as underpinning this hypothesis. While our data make the case that other mechanisms override an initial increase in gut permeability, which has been associated with insulin resistance and obesity, to induce weight loss and improve glucose tolerance after bariatric surgery, we are unable to validate this hypothesis at this point.

Finally, the reported correlations on bacterial quantity and those related to bacterial composition with low effect size should be considered with caution. Despite their statistical significance, the biological relevance of these associations might be questionable and commends further independent replications.

Beyond these restrictions and while the evidence for the presence of bacteria in the placenta has been highly controversial, with independent groups refuting these findings, there has been no evidence in the literature that convincingly shows that the blood is a tissue 
constantly free from bacteria or bacterial genetic material. The limitations of the several studies linking bacteria in the blood with disease have been addressed in the present work further adding to its strengths: Diligent sterile handling of materials and pre-treatment of lab materials with UV light was employed. We further included several negative controls (PBS for extraction and blank controls for PCR), while further accounting for production line contamination of medical products using the same tubes and medical materials used to draw the blood in our subjects. These negative controls were confirmed in agarose gel but were still sequenced alongside the true samples. Moreover, they were actively used to identify possible contaminants in the dataset, which we then excluded using the same Software, that had helped debunk or at least shake the pertinacious notion of a "placenta microbiome" [79, 80]. We further replicated findings on bacterial load in an independent cohort, which was processed and analyzed independently. Moreover, we could substantiate the associations between host metabolic health with individual taxa by several different statistical methods while accounting for the impact of relevant covariates, which has, to our knowledge, never been done previously. Beyond a mere diagnostic tool for metabolic disease, which can be currently achieved more easily and at a lower cost, the current work underpins the notion that the blood is a putative ecological niche. Despite extensive immune control, it remains dynamic and reflects metabolic health, warranting further contaminant conscious rigorous work to delineate mechanistic and exploitable pathways and targets similar to the application of pasteurized bacteria (e.g., Akkermansia muciniphila) [81-86] for weight loss and metabolic improvement.

\section{Conclusions}

In summary, after reducing and controlling for both contamination and technical biases, we could detect low amounts of bacterial DNA in the blood of patients, from which we were able to derive a metabolic fingerprint from bacterial DNA composition. We could also observe differences in diversity, amount of bacterial DNA, and bacterial composition between subjects with and without T2D, subjects with or without T2D remission or with varying degrees of weight loss response to bariatric surgery. We further substantiate our findings of bacterial quantity increase after bariatric surgery with imaging of live bacteria. The present work lays a stepping stone and encourages rigorous cross-sectional and longitudinal studies in larger cohorts with both diseased and healthy subjects. These studies should optimally expand over various expertise to provide insights into the functionality and potential role of a "circulatory microbiome" in maintaining health and contributing to the onset and progression of disease.

\section{Abbreviations}

ALAT: Alanin-aminotransferase; ARBs: Angiotensin II receptor blockers; ASAT: Aspartat-aminotransferase; BMI: Body mass index; CRP: C-reactive protein; EBL: Excess BMI loss; eGFR: Estimated glomerular filtration rate; FPG: Fasting plasma glucose; GLP1: Glucagon-like peptide 1; HbA1c: Glycated hemoglobin A1c; HDL: High-density lipoprotein; HOMA-IR: Homeostatic model assessment for insulin resistance; LBP: Lipopolysaccharide binding protein; LDL: Low-density lipoprotein; MetS: Metabolic syndrome; NGT: Normal glucose tolerance; PPI: Proton pump inhibitors;

RDA: Redundancy analysis; rRNA: Ribosomal ribonucleic acid; sCD14: Soluble cluster of differentiation 14; T2D: Type 2 diabetes; WHR: Waist to hip ratio

\section{Supplementary Information}

The online version contains supplementary material available at https://doi. org/10.1186/s13073-021-00919-6.

Additional file 1. Contains tables S1-8 and figures S1-10.

Additional file 2. Contains tables S2-8.

\section{Acknowledgements}

We would like to thank Stefanie Walther, Judith Kammer, Charlott Krahnepuhl, and Eileen Bösenberg for their excellent support in recruiting subjects, supervising subjects during the study and aftercare, and organization and coordination of the studies, and sample, as well as diligent data collection. We also thank Stefanie Ziesche for excellent technical assistance in the lab. We would further like to thank all subjects who took part in the study and without whom this research would not have been possible. We acknowledge the Centre for Chemical Microscopy (ProVIS) at the Helmholtz Centre for Environmental Research for using their sample preparation and microscopy facilities. Special thanks to Katja Nerlich for support during in situ hybridization and fluorescence microscopy image acquisition. Additional file 1: Figs. S1 and S3 are created using BioRender. com. We also acknowledge support from Leipzig University for Open Access Publishing.

\section{Authors' contributions}

RC and PK designed the study; RC performed experiments; RC and LM analyzed the data; NM supervised and conducted CARD-FISH experiments and analyzed related downstream data. NS performed quantification of bacterial cells after CARD-FISH. All authors contributed to manuscript preparation and writing. All authors read and approved the final manuscript.

\section{Funding}

This work was supported by grants from the Deutsche

Forschungsgemeinschaft (DFG, German Research Foundation - Projektnummer 209933838 - SFB 1052; B01, B03, B09) and from IFB AdiposityDiseases (AD2060E, AD2-06E95, and AD2-K7-117). IFB Adiposity Diseases is supported by the Federal Ministry of Education and Research (BMBF), Germany, FKZ: 01EO1501. RC was supported by a junior research grant by the Medical Faculty, University of Leipzig, and by the Federal Ministry of Education and Research (BMBF), Germany, FKZ: $01 E 01501$ (IFB AdiposityDiseases, MetaRot program). The content of this work is the responsibility of the authors and does not represent the official views of the funding agencies. Open Access funding enabled and organized by Projekt DEAL.

\section{Availability of data and materials}

The data and code that support the findings of this study are available under "figshare" with the following identifiers:

https://doi.org/10.6084/m9.figshare.12885260

https://doi.org/10.6084/m9.figshare.12876335 $[38,41]$

\section{Declarations}

\section{Ethics approval and consent to participate}

This study was approved by the ethics committee of the University of Leipzig (application number: 047-13-28012013) and all participants involved gave written informed consent. The research was performed in accordance with the principles of the Declaration of Helsinki. 


\section{Consent for publication}

Not applicable.

\section{Competing interests}

The authors declare that they have no competing interests.

\section{Author details}

'Medical Department III - Endocrinology, Nephrology, Rheumatology, University of Leipzig Medical Center, Leipzig, Germany. ${ }^{2}$ Department of Medicine (H7), Karolinska Institutet, C2-94, Karolinska University Hospital, Stockholm, Sweden. ${ }^{3}$ German Centre for Integrative Biodiversity Research (iDiv) Halle-Jena-Leipzig, Leipzig, Germany. ${ }^{4}$ Helmholtz Centre for Environmental Research GmbH - UFZ, Halle, Germany. ${ }^{5}$ Department of Isotope Biogeochemistry, Helmholtz Centre for Environmental Research UFZ, Leipzig, Germany. ${ }^{6}$ Department of Computer Science and Interdisciplinary Center for Bioinformatics, University of Leipzig, Leipzig, Germany. ${ }^{7}$ Department of Visceral, Transplantation, Thoracic and Vascular Surgery, Section of Bariatric Surgery, Leipzig University Hospital, Leipzig, Germany. ${ }^{8} \mathrm{Helmholtz}$ Institute for Metabolic, Obesity and Vascular Research (HI-MAG) of the Helmholtz Zentrum München at the University of Leipzig and University Hospital Leipzig, Leipzig, Germany. ${ }^{9}$ Deutsches Zentrum für Diabetesforschung, Neuherberg, Germany.

\section{Received: 7 April 2021 Accepted: 2 June 2021}

Published online: 22 June 2021

\section{References}

1. Turnbaugh PJ, Ley RE, Mahowald MA, Magrini V, Mardis ER, Gordon Jl. An obesity-associated gut microbiome with increased capacity for energy harvest. Nature. 2006;444(7122):1027-31. https://doi.org/10.1038/na ture05414.

2. Sommer F, Bäckhed F. The gut microbiota--masters of host development and physiology. Nat Rev Microbiol. 2013;11(4):227-38. https://doi.org/10.103 8/nrmicro2974.

3. Karlsson F, Tremaroli V, Nielsen J, Bäckhed F. Assessing the human gut microbiota in metabolic diseases. Diabetes. 2013;62(10):3341-9. https://doi. org/10.2337/db13-0844.

4. Zhang X, Shen D, Fang Z, Jie Z, Qiu X, Zhang C, et al. Human gut microbiota changes reveal the progression of glucose intolerance. Plos One. 2013;8(8):e71108. https://doi.org/10.1371/journal.pone.0071108.

5. Cani PD, Amar J, Iglesias MA, Poggi M, Knauf C, Bastelica D, et al. Metabolic endotoxemia initiates obesity and insulin resistance. Diabetes. 2007;56(7): 1761-72. https://doi.org/10.2337/db06-1491

6. Le Chatelier E, Nielsen T, Qin J, Prifti E, Hildebrand F, Falony G, et al. Richness of human gut microbiome correlates with metabolic markers. Nature. 2013;500(7464):541-6. https://doi.org/10.1038/nature12506.

7. Cotillard A, Kennedy SP, Kong LC, Prifti E, Pons N, Le Chatelier E, et al. Dietary intervention impact on gut microbial gene richness. Nature. 2013; 500(7464):585-8. https://doi.org/10.1038/nature12480.

8. Vandeputte D, Kathagen G, D'hoe K, Vieira-Silva S, Valles-Colomer M, Sabino J, et al. Quantitative microbiome profiling links gut community variation to microbial load. Nature. 2017;551(7681):507-11. https://doi.org/10.1038/na ture24460.

9. Zhang H, DiBaise JK, Zuccolo A, Kudrna D, Braidotti M, Yu Y, et al. Human gut microbiota in obesity and after gastric bypass. Proc Natl Acad Sci U S A 2009;106(7):2365-70. https://doi.org/10.1073/pnas.0812600106.

10. Furet J-P, Kong L-C, Tap J, Poitou C, Basdevant A, Bouillot J-L, et al. Differential adaptation of human gut microbiota to bariatric surgeryinduced weight loss: links with metabolic and low-grade inflammation markers. Diabetes. 2010;59(12):3049-57. https://doi.org/10.2337/db10-0253.

11. Graessler J, Qin $Y$, Zhong $H$, Zhang J, Licinio J, Wong M-L, et al. Metagenomic sequencing of the human gut microbiome before and after bariatric surgery in obese patients with type 2 diabetes: correlation with inflammatory and metabolic parameters. Pharmacogenomics J. 2013;13(6): 514-22. https://doi.org/10.1038/tpj.2012.43.

12. McLaughlin RW, Vali H, Lau PCK, Palfree RGE, de Ciccio A, Sirois M, et al. Are there naturally occurring pleomorphic bacteria in the blood of healthy humans? J Clin Microbiol. 2002;40(12):4771-5. https://doi.org/10.1128/jcm.4 0.12.4771-4775.2002.

13. Anhê FF, Jensen BAH, Varin TV, Servant F, van Blerk S, Richard D, et al. Type 2 diabetes influences bacterial tissue compartmentalisation in human obesity. Nat Metab. 2020;2(3):233-42. https://doi.org/10.1038/s42255-020-01 78-9.

14. Massier L, Chakaroun R, Tabei S, Crane A, Didt KD, Fallmann J, et al. Adipose tissue derived bacteria are associated with inflammation in obesity and type 2 diabetes. Gut. 2020;69(10):1796-806. https://doi.org/10.1136/gutjnl-201 9-320118.

15. Amar J, Serino M, Lange C, Chabo C, lacovoni J, Mondot S, et al. Involvement of tissue bacteria in the onset of diabetes in humans: evidence for a concept. Diabetologia. 2011;54(12):3055-61. https://doi.org/10.1007/ s00125-011-2329-8.

16. Qiu J, Zhou H, Jing Y, Dong C. Association between blood microbiome and type 2 diabetes mellitus: a nested case-control study. J Clin Lab Anal. 2019; 33(4):e22842. https://doi.org/10.1002/jcla.22842.

17. Amar J, Lange C, Payros G, Garret C, Chabo C, Lantieri O, et al. Blood microbiota dysbiosis is associated with the onset of cardiovascular events in a large general population: the D.E.S.I.R. study. Plos One. 2013;8:e54461. https://doi.org/10.1371/journal.pone.0054461.

18. Schierwagen R, Alvarez-Silva C, Madsen MSA, Kolbe CC, Meyer C, Thomas D, et al. Circulating microbiome in blood of different circulatory compartments. Gut. 2019;68(3):578-80. https://doi.org/10.1136/gutjnl-201 8-316227.

19. Sato J, Kanazawa A, Ikeda F, Yoshihara T, Goto H, Abe H, et al. Gut dysbiosis and detection of "live gut bacteria" in blood of Japanese patients with type 2 diabetes. Diabetes Care. 2014;37(8):2343-50. https://doi.org/10.2337/ dc13-2817.

20. Lelouvier B, Servant F, Païssé S, Brunet A-C, Benyahya S, Serino M, et al. Changes in blood microbiota profiles associated with liver fibrosis in obese patients: a pilot analysis. Hepatology. 2016;64(6):2015-27. https://doi.org/1 0.1002/hep.28829

21. Poore GD, Kopylova E, Zhu Q, Carpenter C, Fraraccio S, Wandro S, et al. Microbiome analyses of blood and tissues suggest cancer diagnostic approach. Nature. 2020;579(7800):567-74. https://doi.org/10.1038/s41586-02 0-2095-1.

22. Ortiz S, Zapater $P$, Estrada JL, Enriquez $P$, Rey M, Abad A, et al. Bacterial DNA translocation holds increased insulin resistance and systemic inflammatory levels in morbid obese patients. J Clin Endocrinol Metab. 2014;99(7):257583. https://doi.org/10.1210/jc.2013-4483.

23. Salter SJ, Cox MJ, Turek EM, Calus ST, Cookson WO, Moffatt MF, et al. Reagent and laboratory contamination can critically impact sequence-based microbiome analyses. BMC Biol. 2014;12(1):87. https://doi.org/10.1186/s1291 5-014-0087-z.

24. 2. Classification and diagnosis of diabetes: standards of medical care in diabetes-2020. Diabetes Care. 2020;43(Supplement 1):S14-31. https://doi. org/10.2337/dc20-S002.

25. Whelton PK, Carey RM, Aronow WS, Casey DE, Collins KJ, Dennison Himmelfarb C, et al. 2017 ACC/AHA/AAPA/ABC/ACPM/AGS/APhA/ASH/ ASPC/NMA/PCNA guideline for the prevention, detection, evaluation, and management of high blood pressure in adults: executive summary: a report of the American College of Cardiology/American Heart Association Task Force on Clinical Practice Guidelines. Circulation. 2018;138(17):e426-83. https://doi.org/10.1161/CIR.0000000000000597.

26. Catapano AL, Graham I, de Backer G, Wiklund O, Chapman MJ, Drexel H, et al. 2016 ESC/EAS guidelines for the management of dyslipidaemias: The Task Force for the Management of Dyslipidaemias of the European Society of Cardiology (ESC) and European Atherosclerosis Society (EAS) Developed with the special contribution of the European Association for Cardiovascular Prevention \& Rehabilitation (EACPR). Atherosclerosis. 2016;253:281-344. https://doi.org/10.1016/j.atherosclerosis.2016.08.018.

27. Alberti KGMM, Zimmet P, Shaw J. The metabolic syndrome--a new worldwide definition. Lancet. 2005:366(9491):1059-62. https://doi.org/10.101 6/S0140-6736(05)67402-8.

28. Dirksen C, Jørgensen NB, Bojsen-Møller KN, Kielgast U, Jacobsen SH, Clausen $T R$, et al. Gut hormones, early dumping and resting energy expenditure in patients with good and poor weight loss response after Roux-en-Y gastric bypass. Int J Obes (Lond). 2013;37(11):1452-9. https://doi.org/10.1038/ijo.2 013.15

29. Wallace TM, Levy JC, Matthews DR. Use and abuse of HOMA modeling. Diabetes Care. 2004;27(6):1487-95. https://doi.org/10.2337/diacare.27.6.1487.

30. Sokol H, Pigneur B, Watterlot L, Lakhdari O, Bermúdez-Humarán LG, Gratadoux J-J, et al. Faecalibacterium prausnitzii is an anti-inflammatory commensal bacterium identified by gut microbiota analysis of Crohn 
disease patients. Proc Natl Acad Sci U S A. 2008;105(43):16731-6. https://doi. org/10.1073/pnas.0804812105.

31. Valladares R, Sankar D, Li N, Williams E, Lai K-K, Abdelgeliel AS, et al. Lactobacillus johnsonii N6.2 mitigates the development of type 1 diabetes in BB-DP rats. Plos One. 2010;5(5):e10507. https://doi.org/10.1371/journal. pone.0010507.

32. Livak KJ, Schmittgen TD. Analysis of relative gene expression data using real-time quantitative PCR and the 2(-delta delta $C(T))$ method. Methods. 2001;25(4):402-8. https://doi.org/10.1006/meth.2001.1262.

33. Cole JR, Wang Q, Fish JA, Chai B, McGarrell DM, Sun Y, et al. Ribosomal Database Project: data and tools for high throughput rRNA analysis. Nucleic Acids Res. 2013;42(D1):D633-42. https://doi.org/10.1093/nar/gkt1244.

34. Wickham H. ggplot2: Elegant Graphics for Data Analysis. New York: Springer; 2016. ISBN 978-3-319-24277-4. http://ggplot2.org.

35. Kassambra A. ggpubr: 'ggplot2' Based Publication Ready Plots. R package version 0.2. 2018. https://CRAN.R-project.org/package $=$ ggpubr.

36. Wei T, Simko V. R package "corrplot": Visualization of a Correlation Matrix. (Version 0.89). 2021. https://github.com/taiyun/corrplot.

37. McMurdie PJ, Holmes S. phyloseq: an R package for reproducible interactive analysis and graphics of microbiome census data. Plos One. 2013;8(4): e61217. https://doi.org/10.1371/journal.pone.0061217.

38. Chakaroun RM, Massier L, Heintz-Buschart A, Said N, Fallmann J, Crane A, et al. Circulating bacterial signature is linked to metabolic disease and shifts with metabolic alleviation after bariatric surgery: Internet Resource. https:// figshare.com/articles/online_resource/raw_16S_rRNA_sequences/12876335/1.

39. Ewels P, Magnusson M, Lundin S, Käller M. MultiQC: summarize analysis results for multiple tools and samples in a single report. Bioinformatics. 2016;32(19):3047-8. https://doi.org/10.1093/bioinformatics/btw354.

40. Bolyen E, Rideout JR, Dillon MR, Bokulich NA, Abnet CC, Al-Ghalith GA, et al. Reproducible, interactive, scalable and extensible microbiome data science using QIIME 2. Nat Biotechnol. 2019;37(8):852-7. https://doi.org/10.1038/s41 587-019-0209-9.

41. Chakaroun RM, Massier L, Heintz-Buschart A, Said N, Fallmann J, Crane A, et al. Circulating bacterial signature is linked to metabolic disease and shifts with metabolic alleviation after bariatric surgery: online resource. https://figsha re.com/articles/online_resource/QIIME2_code_and_artifacts/12885260/1.

42. Callahan BJ, McMurdie PJ, Rosen MJ, Han AW, Johnson AJA, Holmes SP. DADA2: high-resolution sample inference from Illumina amplicon data. Nat Methods. 2016;13(7):581-3. https://doi.org/10.1038/nmeth.3869.

43. Katoh K, Misawa K, Kuma K-I, Miyata T. MAFFT: a novel method for rapid multiple sequence alignment based on fast Fourier transform. Nucleic Acids Res. 2002;30(14):3059-66. https://doi.org/10.1093/nar/gkf436.

44. Pedregosa F, Varoquaux G, Gramfort A, Michel V, Thirion B, Grisel O. Scikitlearn: machine learning in Python. http://arxiv.org/abs/1201.0490. Accessed 20 Aug 2020.

45. Quast C, Pruesse E, Yilmaz P, Gerken J, Schweer T, Yarza P, et al. The SILVA ribosomal RNA gene database project: improved data processing and webbased tools. Nucleic Acids Res. 2013;41(D1):D590-6. https://doi.org/10.1093/ nar/gks1219.

46. Davis NM, Proctor DM, Holmes SP, Relman DA, Callahan BJ. Simple statistical identification and removal of contaminant sequences in marker-gene and metagenomics data. Microbiome. 2018;6(1):226. https://doi.org/10.1186/s401 68-018-0605-2.

47. Oksanen J. Vegan: community ecology package. Ordination methods, diversity analysis and other functions for community and vegetation ecologists | World Agroforestry.

48. Leo Lahti, et al. microbiome R package. http://microbiome.github.io.

49. Liaw A, Wiener M. Classification and Regression by randomForest. R News. 2002;2(3):18-22.

50. Robin X, Turck N, Hainard A, Tiberti N, Lisacek F, Sanchez J-C, et al. pROC: an open-source package for $\mathrm{R}$ and $\mathrm{S}+$ to analyze and compare ROC curves. BMC Bioinformatics. 2011;12(1):77. https://doi.org/10.1186/1471-2105-12-77.

51. Evans JSMMA. rfUtilities; 2018.

52. Murphy MA, Evans JS, Storfer A. Quantifying Bufo boreas connectivity in Yellowstone National Park with landscape genetics. Ecology. 2010;91 (1):25261. https://doi.org/10.1890/08-0879.1.

53. Love Ml, Huber W, Anders S. Moderated estimation of fold change and dispersion for RNA-seq data with DESeq2. Genome Biol. 2014;15(12):550. https://doi.org/10.1186/s13059-014-0550-8.

54. Pernthaler A, Pernthaler J. Simultaneous fluorescence in situ hybridization of mRNA and rRNA for the detection of gene expression in environmental microbes. Meth Enzymol. 2005;397:352-71. https://doi.org/10.1016/500766879(05)97021-3.

55. Amann Rl, Krumholz L, Stahl DA. Fluorescent-oligonucleotide probing of whole cells for determinative, phylogenetic, and environmental studies in microbiology. J Bacteriol. 1990;172:762-70.

56. Lakka H-M, Laaksonen DE, Lakka TA, Niskanen LK, Kumpusalo E, Tuomilehto $J$, et al. The metabolic syndrome and total and cardiovascular disease mortality in middle-aged men. JAMA. 2002;288(21):2709-16. https://doi. org/10.1001/jama.288.21.2709.

57. Forner L, Larsen T, Kilian M, Holmstrup P. Incidence of bacteremia after chewing, tooth brushing and scaling in individuals with periodontal inflammation. J Clin Periodontol. 2006;33(6):401-7. https://doi.org/10.1111/ j.1600-051X.2006.00924.X.

58. Amar J, Chabo C, Waget A, Klopp P, Vachoux C, Bermúdez-Humarán LG, et al. Intestinal mucosal adherence and translocation of commensal bacteria at the early onset of type 2 diabetes: molecular mechanisms and probiotic treatment. EMBO Mol Med. 2011;3(9):559-72. https://doi.org/10.1002/emmm.201100159.

59. Traykova D, Schneider B, Chojkier M, Buck M. Blood microbiome quantity and the hyperdynamic circulation in decompensated cirrhotic patients. Plos One. 2017;12(2):e0169310. https://doi.org/10.1371/journal.pone.0169310.

60. Païssé $S$, Valle $C$, Servant F, Courtney M, Burcelin R, Amar J, et al. Comprehensive description of blood microbiome from healthy donors assessed by 165 targeted metagenomic sequencing. Transfusion. 2016;56(5): 1138-47. https://doi.org/10.1111/trf.13477.

61. Whittle E, Leonard MO, Harrison R, Gant TW, Tonge DP. Multi-method characterization of the human circulating microbiome. Front Microbiol. 2018;9:3266. https://doi.org/10.3389/fmicb.2018.03266.

62. Moriyama K, Ando C, Tashiro K, Kuhara S, Okamura S, Nakano S, et al. Polymerase chain reaction detection of bacterial 16S rRNA gene in human blood. Microbiol Immunol. 2008;52(7):375-82. https://doi.org/10.1111/j.13480421.2008.00048.x.

63. Dinakaran V, Rathinavel A, Pushpanathan M, Sivakumar R, Gunasekaran P, Rajendhran J. Elevated levels of circulating DNA in cardiovascular disease patients: metagenomic profiling of microbiome in the circulation. Plos One. 2014;9(8):e105221. https://doi.org/10.1371/journal.pone.0105221.

64. Olde Loohuis LM, Mangul S, Ori APS, Jospin G, Koslicki D, Yang HT, et al. Transcriptome analysis in whole blood reveals increased microbial diversity in schizophrenia. Transl Psychiatry. 2018;8(1):96. https://doi.org/10.1038/s413 98-018-0107-9.

65. Maier L, Pruteanu M, Kuhn M, Zeller G, Telzerow A, Anderson EE, et al. Extensive impact of non-antibiotic drugs on human gut bacteria. Nature. 2018;555(7698):623-8. https://doi.org/10.1038/nature25979.

66. Arumugam M, Raes J, Pelletier E, Le Paslier D, Yamada T, Mende DR, et al. Enterotypes of the human gut microbiome. Nature. 2011;473(7346):174-80. https://doi.org/10.1038/nature09944.

67. Qin J, Li Y, Cai Z, Li S, Zhu J, Zhang F, et al. A metagenome-wide association study of gut microbiota in type 2 diabetes. Nature. 2012; 490(7418):55-60. https://doi.org/10.1038/nature11450.

68. Palleja A, Kashani A, Allin KH, Nielsen T, Zhang C, Li Y, et al. Roux-en-Y gastric bypass surgery of morbidly obese patients induces swift and persistent changes of the individual gut microbiota. Genome Med. 2016; 8(1):67. https://doi.org/10.1186/s13073-016-0312-1.

69. Koren O, Spor A, Felin J, Fåk F, Stombaugh J, Tremaroli V, et al. Human oral, gut, and plaque microbiota in patients with atherosclerosis. Proc Natl Acad Sci U S A. 2010;108(Supplement_1):4592-8. https://doi.org/10.1073/pnas.1 011383107.

70. Yoshida N, Yamashita T, Hirata K-i. Gut microbiome and cardiovascular diseases. Diseases 2018. doi:https://doi.org/10.3390/diseases6030056

71. Forslund K, Hildebrand F, Nielsen T, Falony G, Le Chatelier E, Sunagawa S, et al. Disentangling type 2 diabetes and metformin treatment signatures in the human gut microbiota. Nature. 2015;528(7581):262-6. https://doi.org/1 $0.1038 /$ nature 15766

72. Blanchard C, Moreau F, Chevalier J, Ayer A, Garcon D, Arnaud L, et al. Sleeve gastrectomy alters intestinal permeability in diet-induced obese mice. Obes Surg. 2017;27(10):2590-8. https://doi.org/10.1007/s11695-017-2670-1.

73. Aron-Wisnewsky J, Doré J, Clement K. The importance of the gut microbiota after bariatric surgery. Nat Rev Gastroenterol Hepatol. 2012;9(10):590-8. https://doi.org/10.1038/nrgastro.2012.161.

74. Chakaroun RM, Massier L, Kovacs P. Gut microbiome, intestinal permeability, and tissue bacteria in metabolic disease: perpetrators or bystanders? Nutrients. 2020;12(4). https://doi.org/10.3390/nu12041082. 
75. Lockhart PB, Brennan MT, Sasser HC, Fox PC, Paster BJ, Bahrani-Mougeot FK. Bacteremia associated with toothbrushing and dental extraction. Circulation. 2008;117(24):3118-25. https://doi.org/10.1161/CIRCULATIONAHA.107.758524.

76. Parahitiyawa NB, Jin LJ, Leung WK, Yam WC, Samaranayake LP. Microbiology of odontogenic bacteremia: beyond endocarditis. Clin Microbiol Rev. 2009; 22(1):46-64. https://doi.org/10.1128/CMR.00028-08.

77. Schmidt TS, Hayward MR, Coelho LP, Li SS, Costea PI, Voigt AY, et al. Extensive transmission of microbes along the gastrointestinal tract. Elife. 2019;8. https://doi.org/10.7554/eLife.42693.

78. Geraldo MSP, Fonseca FLA, MRdFV G, Feder D. The use of drugs in patients who have undergone bariatric surgery. Int I Gen Med. 2014;7:219-24. https://doi.org/10.2147/IJGM.S55332.

79. de Goffau MC, Lager S, Sovio U, Gaccioli F, Cook E, Peacock SJ, et al. Human placenta has no microbiome but can contain potential pathogens. Nature. 2019:572(7769):329-34. https://doi.org/10.1038/s41586-019-1451-5.

80. Davis NM, Proctor DM, Holmes SP, Relman DA, Callahan BJ. Simple statistical identification and removal of contaminant sequences in marker-gene and metagenomics data. Microbiome. 2018;6(1):226. https://doi.org/10.1186/s401 68-018-0605-2.

81. Cani PD, Vos WM de. Next-generation beneficial microbes: the case of Akkermansia muciniphila. Front Microbiol. 2017;8:1765. doi:https://doi.org/1 0.3389/fmicb.2017.01765.

82. Depommier C, Everard A, Druart C, Plovier H, van Hul M, Vieira-Silva S, et al. Supplementation with Akkermansia muciniphila in overweight and obese human volunteers: a proof-of-concept exploratory study. Nat Med. 2019; 25(7):1096-103. https://doi.org/10.1038/s41591-019-0495-2.

83. Druart C, Plovier H, Hul M, Brient A, Phipps KR, Vos WM de, Cani PD. Toxicological safety evaluation of pasteurized Akkermansia muciniphila. J Appl Toxicol 2020. doi:https://doi.org/10.1002/jat.4044

84. Hänninen A, Toivonen R, Pöysti S, Belzer C, Plovier H, Ouwerkerk JP, et al. Akkermansia muciniphila induces gut microbiota remodelling and controls islet autoimmunity in NOD mice. Gut. 2018;67(8):1445-53. https://doi.org/1 0.1136/gutjnl-2017-314508.

85. Katiraei S, de Vries MR, Costain AH, Thiem K, Hoving LR, van Diepen JA, et al. Akkermansia muciniphila exerts lipid-lowering and immunomodulatory effects without affecting neointima formation in hyperlipidemic APOE*3-Leiden.CETP mice. Mol Nutr Food Res. 2020;64(15): e1900732. https://doi.org/10.1002/mnfr.201900732.

86. Plovier H, Everard A, Druart C, Depommier C, Hul M, Geurts L, et al. A purified membrane protein from Akkermansia muciniphila or the pasteurized bacterium improves metabolism in obese and diabetic mice. Nat Med. 2017;23(1):107-13. https://doi.org/10.1038/nm.4236.

\section{Publisher's Note}

Springer Nature remains neutral with regard to jurisdictional claims in published maps and institutional affiliations.

Ready to submit your research? Choose BMC and benefit from:

- fast, convenient online submission

- thorough peer review by experienced researchers in your field

- rapid publication on acceptance

- support for research data, including large and complex data types

- gold Open Access which fosters wider collaboration and increased citations

- maximum visibility for your research: over $100 \mathrm{M}$ website views per year

At $\mathrm{BMC}$, research is always in progress.

Learn more biomedcentral.com/submissions 